\section{Heterologous expression of bacterial natural product biosynthetic pathways $\uparrow$}

Cite this: DOI: $10.1039 / \mathrm{c} 8 \mathrm{np00091c}$

\author{
Liujie Huo, (D) a Joachim J. Hug, (DD ${ }^{\mathrm{b}}$ Chengzhang Fu, (D) ${ }^{\mathrm{b}}$ Xiaoying Bian, (D) *a \\ Youming Zhang ${ }^{\star a}$ and Rolf Müller (D)*b
}

Covering: 2013 to June 2018
Heterologous expression of natural product biosynthetic pathways is of increasing interest in microbial biotechnology, drug discovery and optimization. It empowers not only the robust production of valuable biomolecules in more amenable heterologous hosts but also permits the generation of novel analogs through biosynthetic engineering. This strategy also facilitates the discovery of novel bioactive compounds following the functional expression of cryptic biosynthetic gene clusters (BGCs) from fastidious original producers or metagenomic DNA in surrogate hosts, thus facilitating genome mining in the post-genomic era. This review discusses recent advances and trends pertaining to the heterologous production of bacterial natural products, with an emphasis on new techniques, heterologous hosts, and novel chemistry since 2013.

10

\section{Introduction}

2. Technical advances in heterologous expression of BGCs

2.1 Cloning \& reconstruction of BGCs

2.2 Considerable factors affecting heterologous expression

3. Emerging heterologous hosts for natural product production

4. Yield improvement by heterologous expression

5. Identification of biosynthetic pathways for known natural products by heterologous expression

6. Discovery of novel compounds by heterologous expression of BGCs from cultured bacteria

6.1 Expression of cryptic BGCs

6.2 Biosynthetic engineering/combinatorial biosynthesis

7. Discovery of novel compounds by heterologous expression of DNA from uncultured bacteria and metagenomic DNA

8. Conclusion

9. Conflicts of interest

10. Acknowledgements

11. References

${ }^{a}$ Shandong University, Helmholtz Institute of Biotechnology, State Key Laboratory of Microbial Technology, Qingdao 266237, P. R. China. E-mail: bianxiaoying@sdu. edu.cn; zhangyouming@sdu.edu.cn

${ }^{b}$ Department of Microbial Natural Products (MINS), Helmholtz Institute for Pharmaceutical Research Saarland (HIPS), Helmholtz Centre for Infection Research (HZI), Campus E8 1, 66123 Saarbrücken, Germany. E-mail: Rolf.Mueller@ helmholtz-hzi.de
In recent decades, scientists have discovered an enormous number of naturally occurring metabolites exhibiting structural diversity and multifarious bioactivities. ${ }^{1}$ To date, approximately $40 \%$ of clinical drugs are either natural products or derivatives thereof. ${ }^{2,3}$ Associated products have been used as antibiotics, antitumor agents, immunosuppressive agents, insecticides, and herbicides as well as antiparasitic agents. ${ }^{4,5}$ Microorganisms are considered to be one of the most prolific sources of novel natural products for two reasons: (1) historically, microorganisms have been shown to be sustainable and efficient producers for pharmaceutically relevant compounds; (2) only a very small fraction of microorganisms have been exploited for the production of bioactive secondary metabolites; thus there exists great potential for the discovery of novel natural products from previously underexploited prokaryotic hosts.

Microorganisms can produce many classes of secondary metabolites, such as polyketides, peptides, alkaloids, terpenoids and saccharides. In this review, we focus on bacterial natural products, among which polyketides and peptides are the two most abundant types. They are mostly biosynthesized from simple monomeric building blocks including amino acids or short-chain carboxylic acids using giant multifunctional enzymes named non-ribosomal peptide synthetases (NRPSs) ${ }^{6}$ and polyketide synthases (PKSs), ${ }^{7-9}$ respectively. Although the aforementioned enzymes feature differences in utilization of building blocks, activation of substrates and condensation mechanisms, both classes of enzymes share notable similarities 
in relation to the modular architecture of various catalytic domains and assembly-line like mechanisms. Based on the socalled "multiple carrier thiotemplate mechanism", ${ }^{10}$ PKSs and NRPSs exhibit a multimodular organization consisting of repetitive catalytic units called modules. These modules are responsible for the incorporation of singular specific residues into the elongating "ketide" or "peptide" chain. Furthermore, each module can be subdivided into domains, which represent the catalytic subunits in charge of individual biosynthetic steps associated with precursor loading, condensation, optional modifications and the release of finalized products. This model is underpinned by a chain elongation reaction that proceeds via covalently bound substrates, intermediates, and products. In order for these reactions to proceed, each carrier protein (CP) domain must be post-translationally modified by a dedicated phosphopantetheinyl transferase (PPTase) which catalyzes the in trans transfer of a $4^{\prime}$-phosphopantetheine (Ppant) moiety from coenzyme A $(\mathrm{COA})$ to a highly conserved serine residue in the $\mathrm{CP}$; the reaction results in the conversion of the catalytically inactive apo-CP into active holo-CP. The choice of this thioester chemistry for activation of both acyl monomers and aminoacyl monomers provides the thermodynamic driving force and kinetically accessible nucleophiles for the Claisen (in the case of polyketide) or amide bond (in the case of non-ribosomal peptide) condensation. ${ }^{\mathbf{1 1}}$ PPTase has been shown to be carrier protein-specific, ${ }^{12}$ suggesting that the introduction of heterologous or promiscuous PPTases might be necessary for the successful heterologous expression of PKS/NRPS natural product biosynthetic pathways.

Apart from non-ribosomal peptides (NRPs), ribosomally synthesized and post-translationally modified peptides (RiPPs) present another important class of peptide natural product that have become increasingly prevalent as a consequence of recent genome-sequencing efforts. Typically, RiPPs undergo extensive post-translational modifications and, therefore, possess restricted conformational flexibility, which enables more

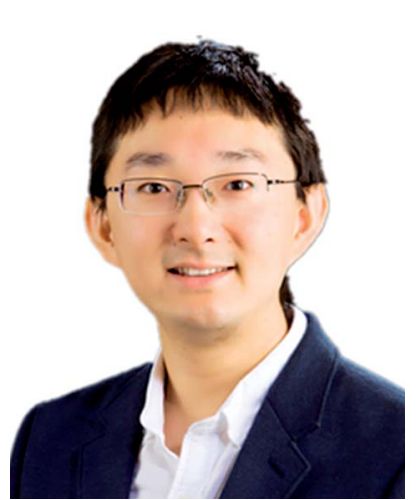

Liujie Huo received his B.Sc. degree (2008) at Tianjin University, China. He obtained his M.Sc. degree (2010) and PhD degree (2014) at Saarland University, Germany, under the supervision of Prof. Rolf Müller. Then he joined Prof. Wilfred A. van der Donk's lab for three-year postdoctoral research at University of Illinois at UrbanaChampaign and Howard Hughes Medical Institute, USA. In 2017 he returned to China as a "Qilu" Youth Scholar Professor at Shandong University. His research focuses on genome mining and genetic engineering of microbial natural products, as well as biochemical studies on interesting biosynthetic tailoring enzymes.
Joachim J. Hug received his B.Sc. degree (2012) and his diploma in pharmacy (2015) at the University of Freiburg in Germany. He carried out his diploma thesis at the University of Cambridge in Professor Peter Leadlay's laboratory and received for his work a diploma award from the German Pharmaceutical Society. In April 2016, he started as a PhD student at Saarland University in Professor Rolf Müller's laboratory. Since 2017, Joachim has been a fellow of the Boehringer Ingelheim Fonds. His research focuses on the biosynthesis of microbial natural products with an emphasis on myxobacteria.

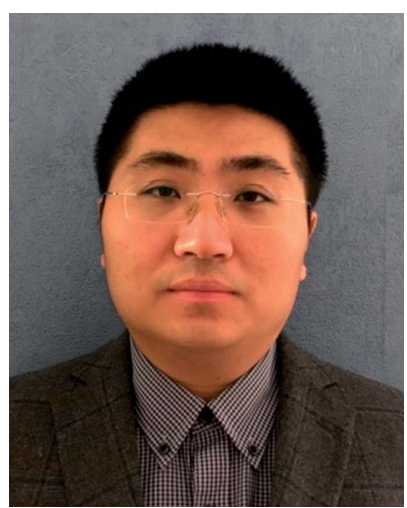

Chengzhang Fu studied biotechnology from 2002 to 2006 at Zhejiang University, China. He received his $P h D$ degree in biochemistry and molecular biology at Institute of Microbiology, Chinese Academy of Sciences in 2011. In 2012, he became Alexander von Humboldt fellow of Professor Rolf Müller at the University of Saarland, Germany. With Professor Müller, he worked on the biosynthesis of natural products produced by actinobacteria and myxobacteria, as well as the mode of action of novel antibiotics. His current research interests are in exploring and engineering biosynthetic gene pathways from bacteria and environmental DNA.

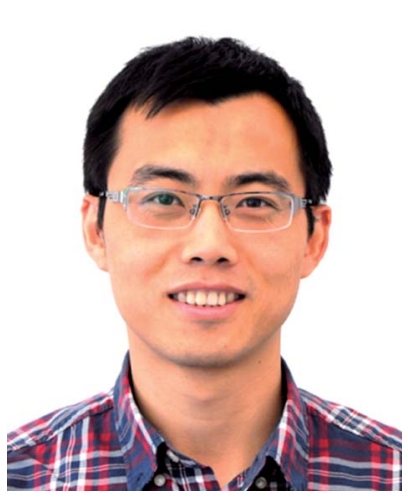

Xiaoying Bian received his B.Sc. (2005) and M.Sc. degree (2008) at Northwest A\&F University, China. He obtained his PhD in 2013 at Saarland University, Germany, under the supervision of Prof. Rolf Müller. After twoyear postdoctoral research in the Helmholtz-Institute for Pharmaceutical Research Saarland (HIPS), he returned to China as a "Qilu" Youth Scholar Professor at Shandong University. His research focuses on the biotechnology and bioengineering of microbial natural product biosynthetic pathways to promote natural product discovery and optimization in the post-genomic era. 
optimal target recognition, metabolic stability, and chemical functionality. Compared to the biosynthetic concept of PKSs and NRPSs, RiPP biosynthesis is initiated by a ribosomally generated precursor peptide encoded by a structure-encoding gene. After the precursor peptide undergoes a variety of posttranslational modifications upon binding to the corresponding post-translationally modifying enzymes, a final proteolytic cleavage of the leader peptide occurs, leading to the conversion of the precursor peptide into the final product, which exhibits great chemical diversity and various biological activities. ${ }^{13-15} \mathrm{~A}$ prerequisite for efficient heterologous expression of RiPP pathways is the adaption of corresponding post-translational modification tailoring enzymes to be functionally expressed in a heterologous host.

Genes encoding these natural products in bacteria are often clustered, which allows the transfer of an entire biosynthetic pathway into an exogenous host for heterologous expression. Heterologous expression has not only facilitated the discovery of novel natural products based on the ever-increasing availability of genome-sequencing data and the bioinformatic annotation of biosynthetic gene clusters (BGCs), but also provides a means for the characterization of biosynthetic pathways in a genetically amenable host. This strategy also enables genetic modifications of such pathways, allowing the generation of various natural product analogs as well as the optimization of production yield. Despite the availability of a number of optimization methodologies in native producers, such as increasing the supply of biosynthetic precursors, the induction of spontaneous mutations, ${ }^{\mathbf{1 6 - 1 8}}$ the insertion of more suitable promoters, ${ }^{\mathbf{1 9 , 2 0}}$ overexpression of positive regulators, ${ }^{21-23}$ disrupting negative regulators ${ }^{24-26}$ etc., a large number of potentially novel natural products have yet to be isolated. With this in mind, mobilization of the corresponding BGCs into a feasible heterologous host has become a valid alternative

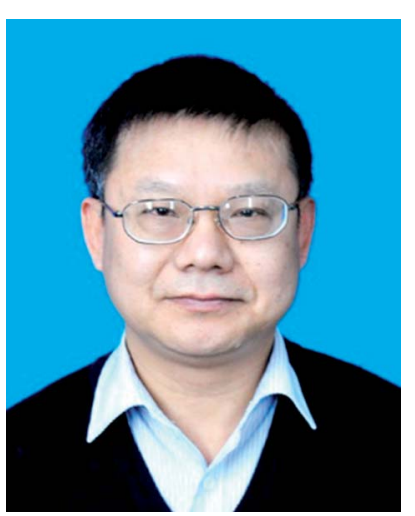

Youming Zhang received his PhD at Ruprecht-Karls-Universität Heidelberg in 1994. After his postdoctoral research in Heidelberg University and European Molecular Biology Laboratory (EMBL), he founded Gene Bridges $\mathrm{GmbH}$ (Germany) in 2000 as the Co-founder and Chief Scientific Officer. In 2013 he was appointed as the director of the State Key Laboratory of Microbial Technology, Shandong University. He became an awardee of the Recruitment Program of Global Experts in China in 2014. His research is dedicated to the development of the Red/ET recombineering, and its application in microbial natural product heterologous expression, humanized animal models, and tumor targeting bacteria. approach to elucidate these 'hidden' metabolites deriving from cryptic BGCs.

This review aims to provide an inspiring insight into strategies for heterologous expression of bacterial biosynthetic pathways producing natural products since our last review in $2013,{ }^{27}$ including an in-depth evaluation of rapidly developing gene-editing techniques for the cloning and modification of BGCs (Fig. 1). We also describe new and/or engineered amenable heterologous hosts as well as a number of representative studies where synthetic biology/ bioengineering methods were employed to improve production titers. Finally, several successful examples of the identification of biosynthetic pathways for known natural products and the discovery of novel compounds by heterologous expression of cryptic BGCs will be discussed. Although we have endeavored to summarize all the natural products that have been discovered, characterized and engineered through heterologous expression to date (Table S1†), we apologize to all authors whose contributions have been missed due to our inevitable negligence.

\section{Technical advances in heterologous expression of BGCs}

The rapid progress of molecular engineering techniques, including CRISPR/Cas along with advances in synthetic biotechnology, promoted the efficient cloning and reconstruction of BGCs. This is the first consideration for successful heterologous expression strategies. Additional factors that influence heterologous expression, including the copy number of BGCs, the integration position in the genome, promoters and native regulatory elements, have also been investigated during the past five years.

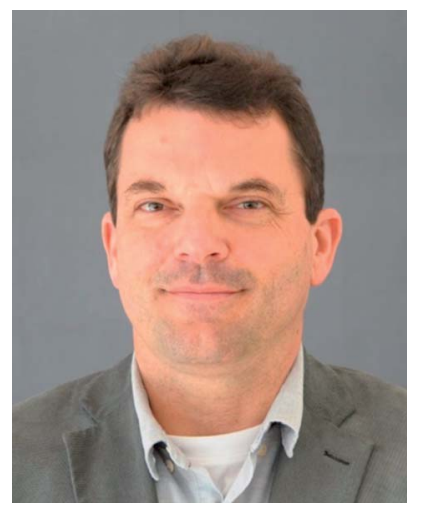

Rolf Müller studied pharmacy in Bonn from 1986-1990 and received his $\mathrm{PhD}$ in 1994. He spent two years as a DFG research scholar at the University of Washington, Seattle and returned to Germany in 1998 as a junior group leader at the former German Research Centre for Biotechnology (GBF). Since October 2003, Rolf has held a chair as professor of pharmaceutical biotechnology at Saarland University. In 2009 he became managing director of the newly founded Helmholtz Institute for Pharmaceutical Research Saarland (HIPS). Rolf is an elected member of German National Academy of Science and Engineering (acatech) and the German National Academy of Sciences Leopoldina. Rolf's research is dedicated to the chemistry and biology of microbial natural products with an emphasis on myxobacteria. 


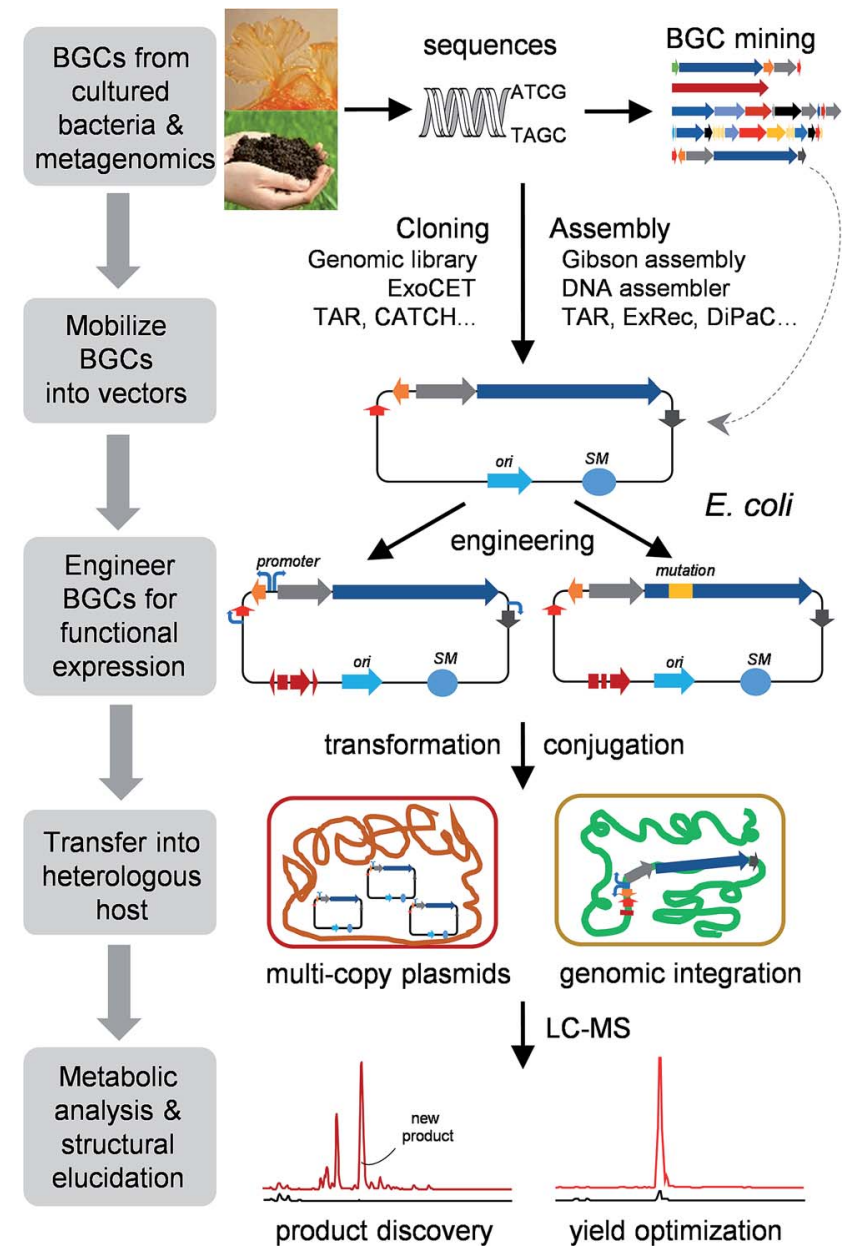

Fig. 1 The general workflow for the heterologous expression of biosynthetic pathways. BGC, biosynthetic gene cluster; ori, origin of replication; SM, selection markers. LC-MS, liquid chromatographmass spectrometry.

\subsection{Cloning \& reconstruction of BGCs}

BGCs of interest are often cloned from native producers using cosmid, fosmid or bacterial artificial chromosome (BAC) libraries. The construction of genomic libraries using BAC vectors is a popular strategy to capture large BGCs (approx. 200 $\mathrm{kb}$ in length), while cosmids generally harbor DNA inserts around the size of $45 \mathrm{~kb}$. Genomic libraries employing P1derived artificial chromosome (PAC) vectors harboring 100$300 \mathrm{~kb}$ DNA segments were also used to clone large chromosomal DNA fragments, including the BGCs of FK506, chaxamycin, vancoresmycin and neoabyssomicin/abyssomicin. ${ }^{28-31}$

Recent progress in relation to genome sequence access and genetic engineering techniques has allowed the direct capture of large-sized BGCs from chromosomal DNA; this has allowed researchers to directly bypass the construction and screening of genomic libraries. Target BGCs can also be cloned into an $E$. coli-Streptomyces BAC conjugation vector, pSBAC, by classical restriction digestion and ligation, such as the cloning of the 95 $\mathrm{kb}$ meridamycin $\mathrm{BGC},{ }^{32}$ or by site-specific recombination of pSBAC into the flanking region followed by digestion and ligation, such as the cloning of $80 \mathrm{~kb}$ tautomycetin $\mathrm{BGC}^{33}$ and the $60 \mathrm{~kb}$ pikromycin BGC. ${ }^{34}$ Restriction digestion combined with Gibson assembly also resulted in obtaining the $41 \mathrm{~kb}$ conglobatin BGC from Streptomyces conglobatus. ${ }^{35}$ A modified Gibson assembly method for cloning large DNA fragments with high GC content antibiotic BGCs from Streptomyces was reported recently. ${ }^{36}$

In 2012, we described linear plus linear homologous recombination (LLHR) mediated by the prophage recombinase RecET to directly clone large BGCs (up to $52 \mathrm{~kb}$ ) from digested genomic DNA in E. coli. ${ }^{37,38}$ The LLHR technique facilitated the rapid cloning of ten BGCs from Photorhabdus luminescens as well as syringolin, glidobactin, edeine, bacillomycin and sevadicin BGCs in a one-step recombination event. ${ }^{37-45}$ To clone the entire $106 \mathrm{~kb}$ salinomycin BGC, three fragments were separately isolated using LLHR and then assembled to form a complete BGC. ${ }^{46}$ Recently, an improved method termed ExoCET (Exonuclease Combined with RecET recombination) was described, combining in vitro exonuclease, e.g. T4 polymerase, and annealing via LLHR to capture a specified region of BGCs ( $>50$ $\mathrm{kb}$ ) from bacterial genomic DNA preparations with nucleotide precision. ${ }^{47}$ ExoCET permitted the direct cloning of the $106 \mathrm{~kb}$ salinomycin BGC into a BAC vector using digested genomic DNA (generated by restriction enzymes or in vitro RNA-guided Cas9 excision) in a single step with an acceptable efficacy. This approach can be used to clone most bacterial BGCs (up to $106 \mathrm{~kb}$ ) for heterologous expression because BGCs typically range from 10 to $120 \mathrm{~kb}$. Strategies deploying CRISPR/Cas9 permit cleavage in vitro and overcome the limitations caused by the availability of unique restriction enzyme sites next to the target BGC to release linear DNA fragments of appropriate size for efficient homologous recombination.

CATCH (Cas9-Assisted Targeting of CHromosome segments) combines in vitro RNA-guided Cas9 cleavage of bacterial chromosomes and Gibson assembly ${ }^{48}$ to clone long genomic sequences (up to $100 \mathrm{~kb}$ ) into cloning vectors. ${ }^{49}$ Indeed, the cloning of a $78 \mathrm{~kb}$ bacillaene BGC from Bacillus subtilis into a BAC vector and of the $36 \mathrm{~kb}$ jadomycin BGC and a $32 \mathrm{~kb}$ chlortetracycline BGC from Streptomyces into the p15A vector revealed the versatility of CATCH in the cloning of large BGCs into vectors with positive rates of approximately $90 \% .^{49}$ The 24 kb BGC of septacidin 1 was cloned using CATCH and successfully expressed in the heterologous host $S$. albus J1074. ${ }^{50}$

Larionov et al. developed a yeast-based transformationassociated recombination (TAR) cloning approach, ${ }^{51-53}$ which has been applied to capture and express large BGCs from environmental DNA (eDNA) samples and bacterial genomes using a BAC-based shuttle capture vector pTARa. ${ }^{54}$ These authors recovered a large number of natural product BGCs from overlapping soil-derived eDNA clones; they subsequently reassembled the resulting BGCs using TAR and directly cloned the colibactin BGC from Citrobacter koseri. ${ }^{54-56}$ Moore's group reported a TAR-based genetic platform for the direct cloning, refactoring and heterologous expression of a "silent" BGC to generate a new antibiotic by construction of a pCAP01 capture vector. A $67 \mathrm{~kb}$ NRPS BGC from the marine actinomycete Saccharomonospora sp. CNQ-490 was successfully captured; this 


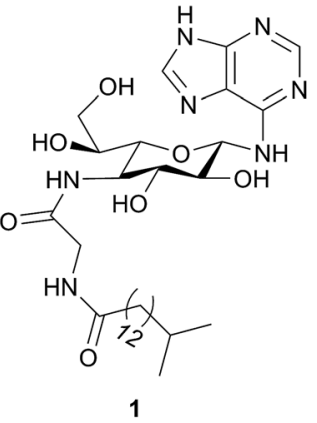

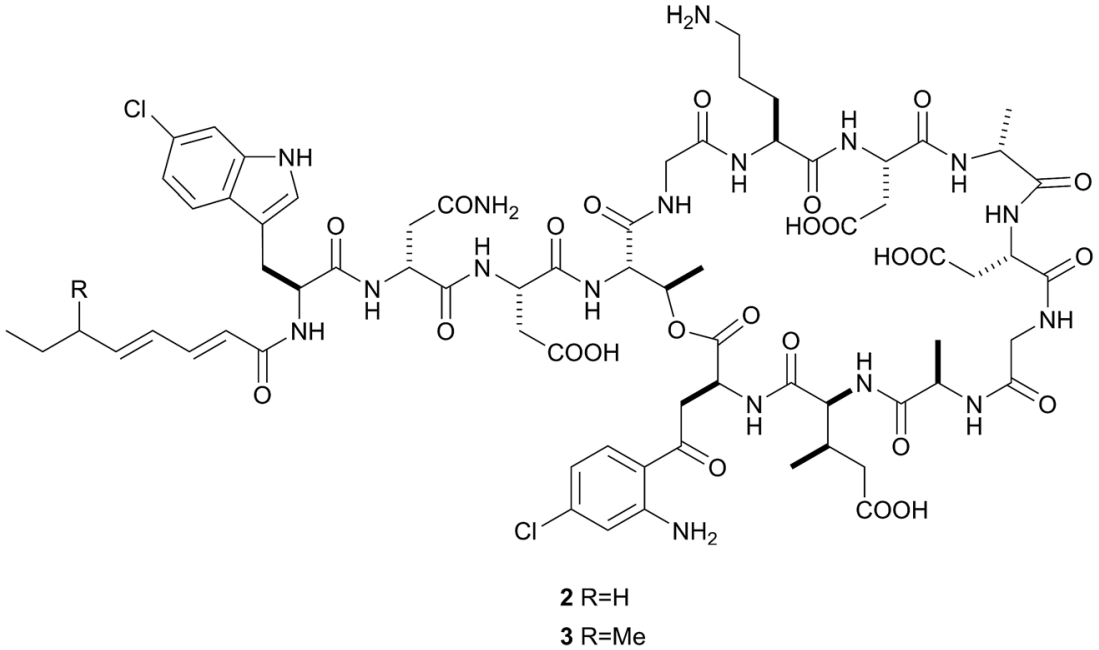

approach led to the discovery of the lipopeptide antibiotic taromycin A 2 and B $3 .^{57,58}$ This platform also facilitated the heterologous expression of the enterocin BGC from the marine actinomycete Salinispora pacifica, ${ }^{59}$ the $34 \mathrm{~kb}$ alterochromide BGC from the marine proteobacterium Pseudoalteromonas piscicida JCM20779 using vector pACR01, ${ }^{60}$ the amicoumacin BGC from the marine Bacillus subtilis 1779 using vector pCAPB2, ${ }^{61}$ the colibactin BGC from E. coli CFT073, ${ }^{62}$ the thiotetronic acid antibiotics thiolactomycin and thiotetroamide BGC from Salinispora, ${ }^{63}$ the nataxazole BGC from Streptomyces sp. Tü 6176, ${ }^{64}$ the 6-demethylchlortetracycline BGC from Streptomyces aureofaciens ${ }^{65}$ the violacein BGC from Pseudoalteromonas luteoviolacea 2 ta16 (ref. 66) and the cosmomycin BGC from Streptomyces sp. CNT-302. ${ }^{67}$ In a separate study, Cas9 digestion along with yeast TAR cloning were used to retrieve long genomic DNA fragments from human genomic DNA. ${ }^{68}$ The latter retrieval strategy was proved to be highly effective and can potentially be used for the isolation and cloning of BGCs.

Du et al. devised a novel strategy for cloning large BGCs in Streptomyces based on phage $\phi$ BT1 integrase-mediated sitespecific recombination. This strategy was used to clone a 25 $\mathrm{kb}$ actinorhodin BGC from $S$. coelicolor M145, a $45 \mathrm{~kb}$ napsamycin BGC and a $157 \mathrm{~kb}$ daptomycin BGC from $S$. roseosporus NRRL 15998 with a reported cloning success frequency greater than $80 \% .{ }^{69}$ This method required two single-crossover steps for the targeted integration of mutated attB and attP sites into the chromosome; thus, a genetic manipulation system of the native producer needs to be established initially prior to heterologous expression.

The assembly of a BGC from several fragments is also achievable. TAR has been used to assemble the grecocycline gene cluster from PCR fragments followed by heterologous expression using a new set of integrative vectors. ${ }^{70}$ The same approach has been used to discover new alpiniamide derivatives through heterologous expression. ${ }^{71}$ Type II polyketide topoisomerase inhibitor pyxidicyclines (B 4) have been uncovered through a self-resistance guided genome mining approach and induced gene expression in the myxobacterial host Pyxidicoccus fallax An d48. The underlying BGC has been assembled and reorganized using the aforementioned PCR-based TAR cloning strategy to produce heterologous pyxidicyclines in the myxobacterial host strains Myxococcus xanthus DK1622 and Stigmatella aurantiaca DW4/3-1. ${ }^{72}$ Heterologous expression of this downregulated gene cluster was facilitated through the exchange of promoters, the reorganization of the operon structure and controlled expression of the self-resistance mechanism, proving the applicability of this approach for these emerging myxobacterial heterologous hosts. Zhao and colleagues employed a DNA assembly method ${ }^{73,74}$ to reconstruct a previously uncharacterized $9.5 \mathrm{~kb}$ polycyclic tetramate macrolactam (PTM) BGC from PCR-generated fragments that were combined with six constitutive promoters upstream of each associated gene. This methodology led to the discovery of three new PTMs following heterologous expression in S. lividans. ${ }^{75}$ 
The Bode group used the "overlap extension PCR-yeast homologous recombination" (ExRec) method to assemble 15 BGCs (up to $45 \mathrm{~kb}$ ) from Photorhabdus and Xenorhabdus. ${ }^{76}$ Direct Pathway Cloning (DiPaC) utilizes long-amplicon PCR and homology-based assembly for the in vitro construction of vector systems for cloning small- to mid-sized BGCs, resulting in the discovery of a new phenazine fontizine A 5 from Serratia fonticola, the heterologous production of anabaenopeptins from Nostoc punctiforme and the transfer of the native erythromycin BGC from Saccharopolyspora erythraea into Streptomyces species. ${ }^{77}$ In addition, DiPaC combined with sequence- and ligation-independent cloning (SLIC) reduces the requisite cloning time and reagent costs, which enabled the successful refactoring of the cyanobacterial $23 \mathrm{~kb}$ BGC and the heterologous production of the depsipeptide hapalosin 6 in $E$. coli. ${ }^{78}$ Small DNA fragments are routinely produced by multiple rounds of PCR or direct chemical synthesis; however, random mutations cannot be excluded entirely. Incorrect pairing of fragments during the assembly process could also cause unexpected mutations or deletions, especially if numerous repeat sequences are present in PKS or NRPS genes. ${ }^{79}$ But a gene synthesis and assembly strategy provides options for optimized codon usage along with the introduction of appropriate regulatory elements. ${ }^{27}$ Thus, this strategy represents an efficient alternative that enables the reconstruction of BGCs for heterologous expression in a distant host with a diverse GC content or regulatory system. But this is also the only method that can be used to acquire BGCs by de novo design for novel natural products.
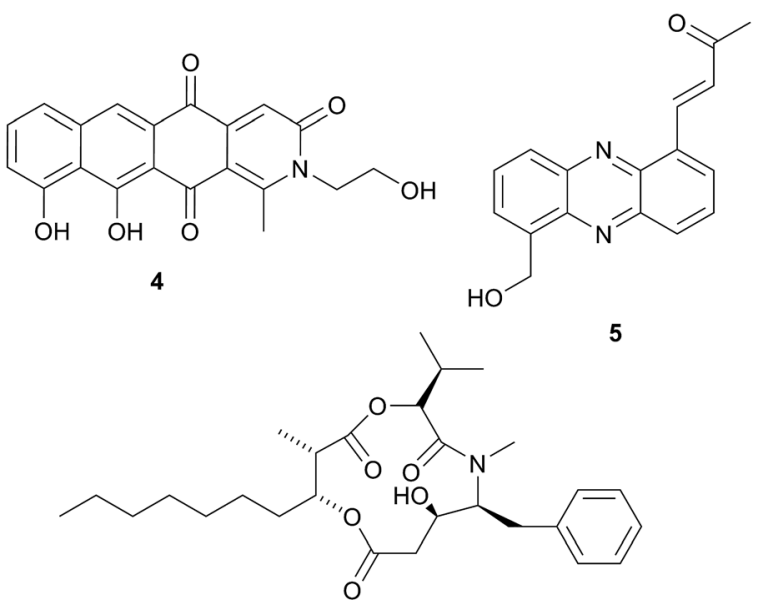

6

These in vivo, in vitro and in vivo/in vitro hybrid strategies, especially methods that employ Cas9 cleavage, greatly accelerate the speed of cloning or assembly of BGCs for heterologous expression. It is hoped that these methodologies will greatly improve genome mining efforts that precede the discovery of novel compounds and yield improvements. A collective example representing the emerging approaches applied in cloning/ assembly of large BGCs for heterologous expression is summarized in Table 1.

\subsection{Considerable factors affecting heterologous expression}

To ensure the robust expression of BGCs in a heterologous host, gene-controlling elements, such as promoters, regulatory factors, determinants of copy number of BGC or the BGC positioning in the genome, are important for fine-tuning gene expression.

The native regulatory elements that are naturally associated with a specific gene cluster might not be recognized by the heterologous host. This problem can be circumvented by expressing the cluster in a more closely related heterologous host. However, a recent study suggested that heterologous production of natural products can be influenced by specific host regulatory and/or biosynthetic elements, challenging the hypothesis that host phylogeny is an accurate predictor of host compatibility. ${ }^{6}$ Promoters are the key regulators of gene expression. Prior to the expression of BGCs in a phylogenetically distant host, the native promoter is usually replaced with a suitable promoter for the heterologous host. An example showing that the replacement of the native promoter leads to the successful heterologous expression of a phylogenetically distant cyanobacterial BGC (deriving from Microcystis aeruginosa PCC 7806) is highlighted by the heterologous production of microcystin-LR and [D-Asp ${ }^{3}$ ] microcystin-LR in E. coli GB05MtaA. ${ }^{80}$ A panel of native strong constitutive promoters was identified from Streptomyces albus by RNA-seq and reporter gene experiments. Five of them were used to successfully activate a cryptic PTM BGC in three widely used streptomycetes. The associated promoters enabled higher expression levels compared with previously identified promoters. ${ }^{81}$ Meanwhile, Li et al. took advantage of transcriptome data from the model strain $S$. coelicolor to perform an experimental evaluation. The results suggested the presence of 166 putative constitutive promoters in the genus Streptomyces. Four of the evaluated promoters were used to trigger the expression of a cryptic gene cluster responsible for biosynthesis of jadomycin B, suggesting that these constitutive promoters exhibit a broad spectrum of transcriptional activity and might be valuable tools in metabolic engineering and synthetic biology ${ }^{82}$ Synthetic promoters do not interfere with the regulatory network of the host and might be beneficial for heterologous expression. Siegl et al. designed and constructed a synthetic promoter library based on the -10 and -35 consensus sequences of the constitutive and widely used ermEp1 promoter for fine-tuned gene expression in four actinomycete strains. ${ }^{83}$ Recently, a comprehensive set of ribosomal binding sites (RBSs), terminators and inducible promoters to drive BGC-controlled expression in streptomycetes has been constructed and fully characterized, ${ }^{\mathbf{8 4}-\mathbf{8 6}}$ allowing the refactoring of the antibiotic bottromycin transcriptional gene cluster by replacing the native promoters with randomly generated constitutive synthetic promoters and expressing them in appropriate hosts. The resulting variants of the bottromycin BGC showed 5-50 fold higher titers of bottromycin A2 7, leading to the identification of several new bottromycin derivatives. ${ }^{87} \mathrm{~A}$ series of native or synthetic promoters and RBS in Streptomyces were shown by a quantitative method based on flow cytometry at single-cell (protoplast) resolution, to activate the associated 
Table 1 Selected emerging approaches for cloning and assembly of large BGC for heterologous expression

\begin{tabular}{ll}
\hline Approach & Principle \\
\hline LEXAS & BAC library construction \\
$\begin{array}{l}\text { Restriction digestion and } \\
\text { ligation }\end{array}$ & $\begin{array}{l}\text { PCR amplification and endonuclease enzyme, } \\
\text { ligase }\end{array}$
\end{tabular}

10

Gibson assembly

LLHR (+LCHR)

15

ExoCET

CATCH

TAR cloning

Phage $\phi \mathrm{BT} 1$

integrase-mediated

site-specific recombination

DNA assembler

ExRec

DiPaC
Exonuclease, DNA polymerase, and DNA ligase

RecET-mediated linear-linear homologous recombination for cloning and Red $\alpha \beta$-mediated linear-circular homologous recombination for assembly

In vitro exonuclease ( $\mathrm{T} 4$ polymerase) and LLHR RNA-guided Cas9 nuclease excision and Gibson assembly

In vivo homologous recombination of Saccharomyces cerevisiae
Application examples and references

Streptothricin, ${ }^{162}$ borrelidin, ${ }^{162}$ 8D1-1, 8D1-2 (ref. 162)

2,4-Diacetylphloroglucinol, ${ }^{194}$ enterocin A, ${ }^{195}$ lactococcin $\mathrm{Z},{ }^{196}$ mycosporine-2-(4deoxygadusolyl-ornithine) ${ }^{197}$ mycosporineornithine/mycosporine-lysine, ${ }^{198}$ plantaricyclin $\mathrm{A},{ }^{199}$ thioviridamide, ${ }^{200,201}$ BM1157/BM1300, ${ }^{202}$ clifednamide, ${ }^{153}$ violapyrones ${ }^{164}$

Bicyclomycin, ${ }^{203}$ kocurin, ${ }^{160}$ lasso peptides,${ }^{204}$ thiolactomycin $^{63}$

Bacillomycin, ${ }^{43}$ chuangxinmycin, ${ }^{156}$ fostriecin, ${ }^{205}$ glidobactin, ${ }^{39}$ oxytetracycline,${ }^{127}$ syringolin, ${ }^{45}$ sevadicin, ${ }^{40}$ salinomycin ${ }^{46}$

$106 \mathrm{~kb}$ BGC salinomycin ${ }^{47}$

Septacidin ${ }^{50}$ bacillaene, ${ }^{49}$ jadomycin, ${ }^{49}$ chlortetracycline ${ }^{49}$

Alterochromide ${ }^{60}$ amicoumacin, ${ }^{61}$ cosmomycin, ${ }^{67}$ enterocin, ${ }^{59}$ lyngbyatoxin $\mathrm{A},{ }^{116}$ mithramycin $\mathrm{A},{ }^{128}$ nataxazole,${ }^{64}$ tilivalline,${ }^{206,207}$ taromycin $\mathrm{A},{ }^{57}$ taromycin $\mathrm{B},{ }^{58}$ thiolactomycin, ${ }^{63}$ thiotetroamides, ${ }^{63}$ thiostreptamide S4, ${ }^{208}$ arenimycins $\mathrm{C} / \mathrm{D},{ }^{209}$ arixanthomycins $\mathrm{A},{ }^{187}$ calixanthomycin $\mathrm{A},{ }^{209}$ malacidins ${ }^{184}$

Actinorhodin, ${ }^{69}$ napsamycin, ${ }^{69}$ daptomycin ${ }^{69}$

Two single crossovers for targeted integration of mutated attB and attP into the chromosome, and the $\phi B T 1$ integrase-mediated site-specific recombination excise targeted region PCR or synthesis generated fragments were assembled by yeast in vivo homologous recombination

Overlap extension PCR and in vivo yeast homologous recombination Long-amplicon PCR and HiFi DNA assembly
Polycyclic tetramate macrolactam (SGR810$815)^{75}$

Xenolindicin, ${ }^{76}$ ambactin $^{76}$

Anabaenopeptins, ${ }^{77}$ fontizine $\mathrm{A},{ }^{77}$ hapalosin ${ }^{78}$
BGC and overproduce the cryptic lycopene in a predictable manner in Streptomyces avermitilis. ${ }^{88} \mathrm{Ji}$ et al. constructed a library of constitutively active and synthetic Streptomyces regulatory sequences containing promoters and ribosome binding site regions with diverse transcriptional activities. ${ }^{\mathbf{9 9}}$ These synthetic regulatory sequence libraries will be important in the heterologous expression of BGCs in actinomycetes. This might spawn the discovery of new natural products from downregulated/cryptic BGCs, thereby improving the yield in heterologous hosts.

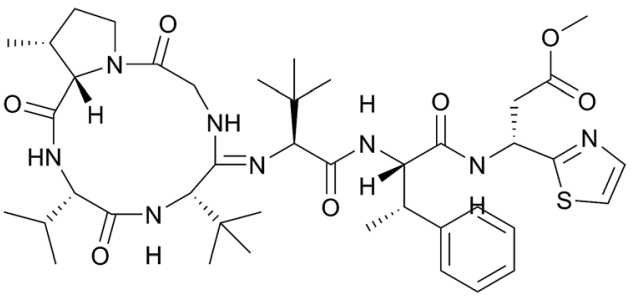

7

One approach to improving the yield of natural products in heterologous hosts necessitates an increased copy number of the related BGC. The Luzhetskyy group reported an approach that increased the product yield of expressed BGCs whereby additional $a t t B$ attachment sites were added to the genome of the heterologous host $S$. albus J1074. When this approach was used to produce pamamycin, didesmethylmensacarcin, griseorhodin A and demethoxyaranciamycinone, the copy number of the respective $\mathrm{BGC}$ increased from one to three in the genome. The yield improvement led to the isolation and identification of a new aranciamycin derivative produced by one of the mutants. $^{90}$ Two copies of the entire pikromycin BGC were introduced in tandem into the heterologous host $S$. lividans by site-specific recombination and homologous recombination. The total production of pikromycin and 10-deoxymethynolide increased twofold compared to the parental strain and 1.2-fold compared with one $S$. lividans strain harboring a single copy of the pikromycin BGC. ${ }^{34} \mathrm{Li}$ et al. performed single-step, multicopy chromosomal integration of target BGCs via the 'one integrase-multiple attB sites' concept. ${ }^{91}$ Several artificial phiC31 $a t t B$ sites were introduced into the heterologous host $S$. coelicolor M1146 and M1152, ${ }^{92}$ such that the yields of chloramphenicol and the antitumor compound YM-216391 were significantly improved (2-23 times) by four-copy chromosomal integration of the corresponding BGCs. ${ }^{91}$ Recently, the Luzhetskyy group introduced additional phage phiC31 attB sites into 
the chromosome of S. albus Del14 in which 15 native BGCs were deleted, allowing integration of up to four copies of a heterologous BGC. One cryptic BGC from Streptomyces spp. and two BGCs from distantly related Frankia spp. strains were successfully activated in these new hosts, leading to the isolation of a new compound, fralnimycin 8, from Frankia alni strain ACN14a. ${ }^{93}$

The Luzhetskyy group also investigated the chromosomal position effect on the heterologous expression of the aranciamycin BGC in $S$. albus $\mathrm{J} 1074$ using a transposon system and a phic31-based vector. The production levels of aranciamycin varied up to eightfold depending on the location of the BGC within the chromosome of S. albus J1074. ${ }^{94}$ Similarly, the $56 \mathrm{~kb}$ antitumor epothilone BGC was randomly integrated into the heterologous host Myxococcus xanthus by transposition. The resulting transformants exhibited a wide range of epothilone production capabilities. Upon sequencing of the associated transcriptome, it was observed that allopatric integrations selectively modified host transcriptomes thereby affecting natural product biosynthesis. ${ }^{95}$ It was observed that the chromosomal location of the integrated heterologous BGC might significantly influence the yield of the produced compound. This result suggests that screening for optimal positions of insertion for one copy of a BGC might be helpful in the future to yield more efficient heterologous hosts.

\section{Emerging heterologous hosts for natural product production}

Heterologous production of natural products can be influenced by specific host regulatory and/or biosynthetic elements; ${ }^{66}$ thus, using a diverse set of heterologous hosts may guarantee more robust heterologous expression. ${ }^{96}$ When factors like growth rate, precursor supply, and self-resistance are considered, actinomycete-derived BGCs are optimally mobilized into engineered streptomycetes, such as S. coelicolor (M1146 or M1152), ${ }^{92}$ S. avermitilis, ${ }^{97}$ S. lividans, ${ }^{98}$ or S. albus $\mathrm{J} 1074$ (or Del14). ${ }^{93,99} \mathrm{M}$. xanthus is currently the best choice for the expression of myxobacterial BGCs, especially for polyketide BGCs. ${ }^{100}$ Bacillus subtilis has generally been used for heterologous expression of firmicute or actinomyces BGCs. ${ }^{101}$ Pseudomonas putida is also a versatile host for the production of natural products, such as myxobacterial myxochromide $S$ and pretubulysin. ${ }^{\mathbf{1 0 2}-104}$ There are also some successful examples in which $E$. coli has been used as a host for the production of complex PKs, NRPs, or RiPPs. ${ }^{105}$ Several emerging heterologous hosts are employed for the production of secondary metabolites from slow-growing and refractory myxobacteria and cyanobacteria.

Myxobacteria are well-known producers of natural products, exhibiting remarkably diverse structures and biological properties. ${ }^{106,107}$ However, only a limited number of heterologous hosts (in particular P. putida and M. xanthus) have been exploited for the heterologous expression of myxobacterial BGCs. ${ }^{108}$ Bian et al. established a novel electroporation procedure for the Burkholderiales strain DSM 7029, which allows large DNA fragments such as the $56 \mathrm{~kb}$ myxobacterial epothilone BGC, to be transformed and randomly transposed into the genome with high efficacy ${ }^{109} \mathrm{~A}$ yield of $307 \mu \mathrm{g} \mathrm{L} \mathrm{L}^{-1}$ of total epothilones (A 9, B 10) was achieved following medium optimization when an exogenous methylmalonyl-CoA pathway and rare tRNA genes were introduced. The strain DSM 7029 exhibits a reduced generation time with a doubling time of $1 \mathrm{~h}$ in contrast to a doubling time of $5 \mathrm{~h}$ for $M$. xanthus, the frequently used heterologous host for myxobacterial BGCs. ${ }^{109}$ Several myxobacterial BGCs, such as vioprolide A 11, ${ }^{110}$ arachazolids and disorazols (Bian, S. C. Wenzel and Müller, unpublished data) have been transferred into strain DSM 7029 for functional expression. Thus, it has been suggested that strain DSM 7029 is potentially an appropriate host for heterologous expression of myxobacterial BGCs. We recently described an efficient genetic engineering system employing native Red recombinases in strain DSM 7029 that was used for a $200 \mathrm{~kb}$ genomic deletion to yield a mutant exhibiting encouraging biomass and growth rate characteristics. ${ }^{111}$ This technical platform facilitates host melioration of strain DSM 7029 by genome reduction of secondary metabolite BGCs, genomic islands, and prophages. The success of these cases in conjunction with the establishment of the highly efficient genome manipulation system further reveals the potential of strain DSM 7029 as an alternative heterologous host for expression of myxobacterial BGCs.

Cyanobacteria are a prolific source of novel bioactive natural products. ${ }^{112,113}$ Efforts to express cyanobacterial BGCs in heterologous hosts including E. coli or Streptomyces sp. have produced variable results. ${ }^{80,114,115}$ Ongley et al. transferred the lyngbyatoxin BGC from an uncultured marine cyanobacterium Moorea producens into $E$. coli, yielding a high-titer production of lyngbyatoxin A $12\left(25.6 \mathrm{mg} \mathrm{L}^{-1}\right)$ and its precursor indolactam $\mathrm{V}$ $\left(150 \mathrm{mg} \mathrm{L}^{-1}\right){ }^{115}$ Videau et al. attempted to develop a genetically tractable Anabaena sp. (PCC 7120). Anabaena is the model freshwater cyanobacterium and it was anticipated that the strain could be a universal heterologous expression host for cyanobacterial BGCs. Heterologous expression of the lyngbyatoxin BGC in Anabaena 7120 using replicative plasmids as BGC carriers led to the production of lyngbyatoxin A with yields which were similar to those in the native producer, Moorea producens. The titer of lyngbyatoxin A can be improved up to 13fold through simple genetic manipulation, showing that Anabaena 7120 could be employed as a model host to investigate the biosynthesis and genetic engineering of cyanobacterial BGCs. ${ }^{116}$ Wang et al. constructed and characterized a promoter library and an RBS library for the expression of heterologous genes in the cyanobacterium Synechocystis sp. PCC 6803, providing a genetic toolbox that can be used to fine-tune gene expression levels and moderate metabolic bottlenecks in cyanobacteria. ${ }^{117}$ Besides genetic engineering steps, attempts to exploit the full biosynthetic capacity of these slow-growing phototrophic organisms are impeded by the requirement for larger amounts of biomass. Guljamow et al. utilized a high-density cultivation approach of Nostoc punctiforme ( $400 \mathrm{~g}$ wet weight $\mathrm{L}^{-1}$ ) to enable the isolation and structural elucidation of novel variants of anabaenopeptin. ${ }^{118}$ The study of this cell-type-specific expression highlighted major reprogramming of the secondary 


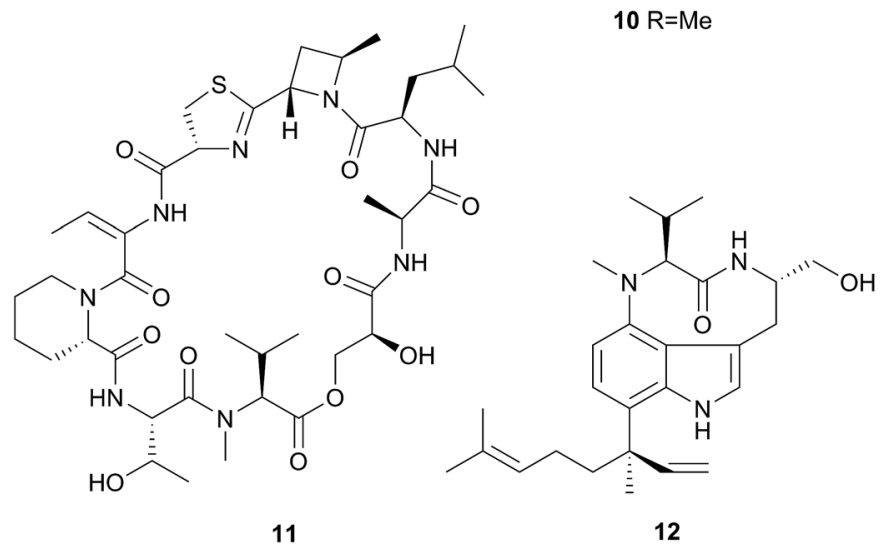

metabolome at high culture density, indicating a crucial prerequisite for heterologous expression in cyanobacterial hosts. Roulet et al. developed the model photosynthetic strain Synechococcus elongatus for the heterologous production of PKSderived compounds. Several modules of essential components of PKS, such as methylmalonyl-CoA formation and PPTase, as well as inducible circuits for the regulated expression of BGC, were designed and introduced into this strain for the biosynthesis of the final PKs. ${ }^{119}$ The mycobacterial PKS-based mycocerosic BGC was expressed in this engineered strain to produce multimethyl-branched esters. This work set the stage for the production of important PKs from mycobacteria or cyanobacteria in a photosynthetic bacterium.

Generally, when bacteria exhibiting genetic stability and a clean metabolic background are supplied with an appropriate amount of biosynthetic precursors, they act as ideal heterologous hosts facilitating the efficient biosynthesis of target natural products. ${ }^{115}$ However, it remains necessary to perform additional genetic engineering steps, such as genome reduction in hosts including $S$. coelicolor ${ }^{92} S$. avermitilis, ${ }^{97,120} S$. albus, ${ }^{93}$ Pseudomonas putida, ${ }^{\mathbf{1 2 1 , 1 2 2}}$ and Corynebacterium glutamicum, ${ }^{\mathbf{1 2 3}}$ for optimal heterologous expression of BGCs. Several comprehensive reviews have summarized the employment of minimized genomes and engineered hosts for the heterologous expression of natural product BGCs. ${ }^{\mathbf{8 1 , 9 6 , 1 2 4 - 1 2 6}}$

\section{Yield improvement by heterologous expression}

Heterologous expression of BGCs in a "superior" host is an important strategy to improve the production of secondary metabolites (Table S1 $\dagger$ ). However, heterologous production often results in low yields due to the differentiation of transcriptional and metabolic regulation, precursor supply, cofactor availability or self-resistance between heterologous and native hosts. Thus, it is important that we rationally engineer BGCs or heterologous hosts to improve the efficiencies of natural product biosynthesis.

The industrial fermentation of oxytetracycline (OTC) by Streptomyces rimosus requires extended periods (8-10 days) to reach optimal titers. To reduce the fermentation time, Yin et al. transferred the OTC BGC into $S$. venezuelae WVR2006, a heterologous host exhibiting advantages such as rapid and dispersed growth, convenience of genetic manipulation, and resistance to OTC. After manipulation of regulatory genes and the supply of malonyl-CoA, the yield of OTC in the heterologous producer reached $431 \mathrm{mg} \mathrm{L}^{-1}$ in a flask after only $48 \mathrm{~h}$, which is comparable to yields from the native producer after 8 days. ${ }^{\mathbf{1 2 7}}$ The mithramycin A BGC was cloned by TAR cloning from Streptomyces argillaceus and transferred into S. lividans TK24 for heterologous production. The yield of mithramycin A 13 in a heterologous host was significantly improved by sequential deletion of known secondary metabolite BGCs, which potentially compete for energy and precursors. Mithramycin A production reached a maximum of approximately $3 \mathrm{~g} \mathrm{~L}^{-1}$ in

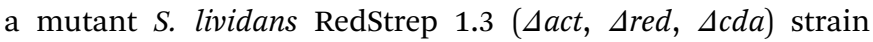
harboring three BGC deletions; this is approximately 20 -fold higher than that observed in the wild-type strain and fivefold higher than that observed in overproducer mutants. ${ }^{128}$ Tan et al. cloned the spinosyn BGC from a BAC library and transferred it into $S$. albus $\mathrm{J} 1074$ resulting in production at an extremely low yield $\left(\sim \mu \mathrm{g} \mathrm{L}^{-1}\right)$. The transcriptional and translational analysis along with metabolomic analysis identified the rate-limiting steps in the heterologous production of spinosyn, guiding the subsequent optimization to overcome these steps. Three highefficiency Streptomyces promoters were used to overexpress rhamnose and forosamine biosynthetic modules, the 
methyltransferase gene and a PKS gene. The resulting production of spinosad (spinosyn A and D) finally reached $1.45 \mathrm{mg} \mathrm{L}^{-1}$, a yield that is approximately 1000-fold higher than that in the original $S$. albus $\mathrm{J} 1074 .{ }^{129}$ This yield is still low in comparison with strains used for industrial applications; however, this transcriptomic-metabolomic-guided BGC modification strategy could be used for the efficient heterologous production of more natural products. The spinosyn BGC was used to substitute the native erythromycin PKS genes in the heterologous host Saccharopolyspora erythraea and spinosad was detected in the resulting recombinants. Metabolic engineering strategies that involved overexpression of several critical biosynthetic genes and the introduction of an additional PPTase encoding the $s f p$ gene as well as UV mutagenesis notably improved the yield of spinosad to a titer of $830 \mathrm{mg} \mathrm{L}^{-1}$, a yield that has shown significant potential in industrial applications. ${ }^{\mathbf{1 3 0}}$ A significant improvement in bottromycin yield was achieved through heterologous expression of refactored corresponding BGC in $S$. lividans. A library of synthetic promoters has been applied, giving a 50-fold improvement in bottromycin production, resulting in the identification of several new derivatives. ${ }^{87}$

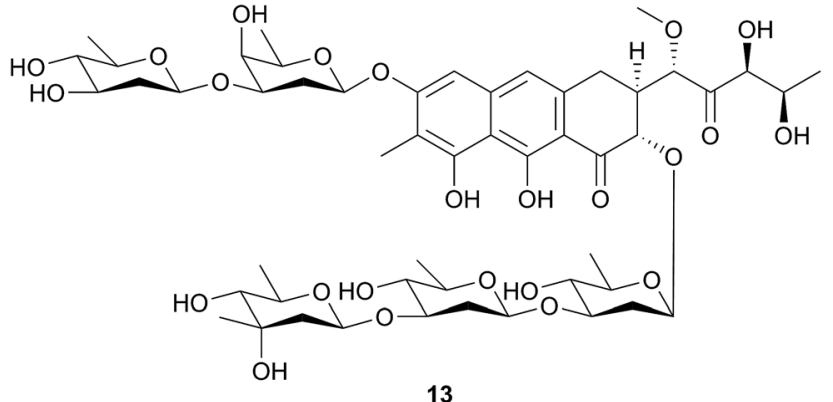

Myxopyronins (A 14, B 15) (MXN) and corallopyronins (A 16, B 17) (COR) are structurally related $\alpha$-pyrone antibiotics produced by myxobacteria. These antibiotics represent a highly promising compound class for the development of antibacterial therapeutic agents, including applications against river blindness. ${ }^{131}$ Sucipto et al. reconstructed MXN and COR BGCs and transferred them independently into the myxobacterial host strain $M$. xanthus DK1622; the yields of MXN and COR were higher than those observed in the native producers. Medium optimization resulted in a significantly higher yield of MXN A (41-fold increase) and COR A (25-fold increase) compared to the standard CTT medium, reaching more than $100 \mathrm{mg} \mathrm{L}^{-\mathbf{1}} \cdot{ }^{\mathbf{1 3 2}}$ The vioprolide (A 11) BGC was also heterologously expressed in $M$. xanthus. The total vioprolide yield can reach $500 \mathrm{mg} \mathrm{L}^{-1}$, an almost 100-fold increase compared to the native producers, following medium optimization in fermenters. ${ }^{\mathbf{1 1 0}}$ Furthermore, medium optimization also resulted in the production time being reduced by $50 \%$. Transfer of the disorazol BGC into $M$. xanthus also resulted in the production of disorazols A1 and A2, while the insertion of a strong promoter upstream of the disD gene to facilitate overexpression of the discrete acyl transferase resulted in a sevenfold increase in disorazol production. ${ }^{\mathbf{1 3 3}}$ The same group combined genetic engineering of the associated biosynthetic pathway from myxobacteria with metabolic engineering of the heterologous host Pseudomonas putida to significantly increase the heterologous expression titer of longchain polyunsaturated fatty acids (LC-PUFA) threefold. ${ }^{\mathbf{1 3 4}}$ The marine myxobacterial antibiotic haliangicin was heterologously produced in $M$. xanthus to increase production 10-fold compared to the native producer Haliangium ochraceum SMP$2 .{ }^{135}$ In addition, this established heterologous platform enabled the investigation of the marine myxobacterial biosynthetic pathway and the production of a bioactive (HeLa $\mathrm{S}_{3}$ cells) unnatural analog through genetic manipulation, which would be more difficult to achieve in the original marine producer.

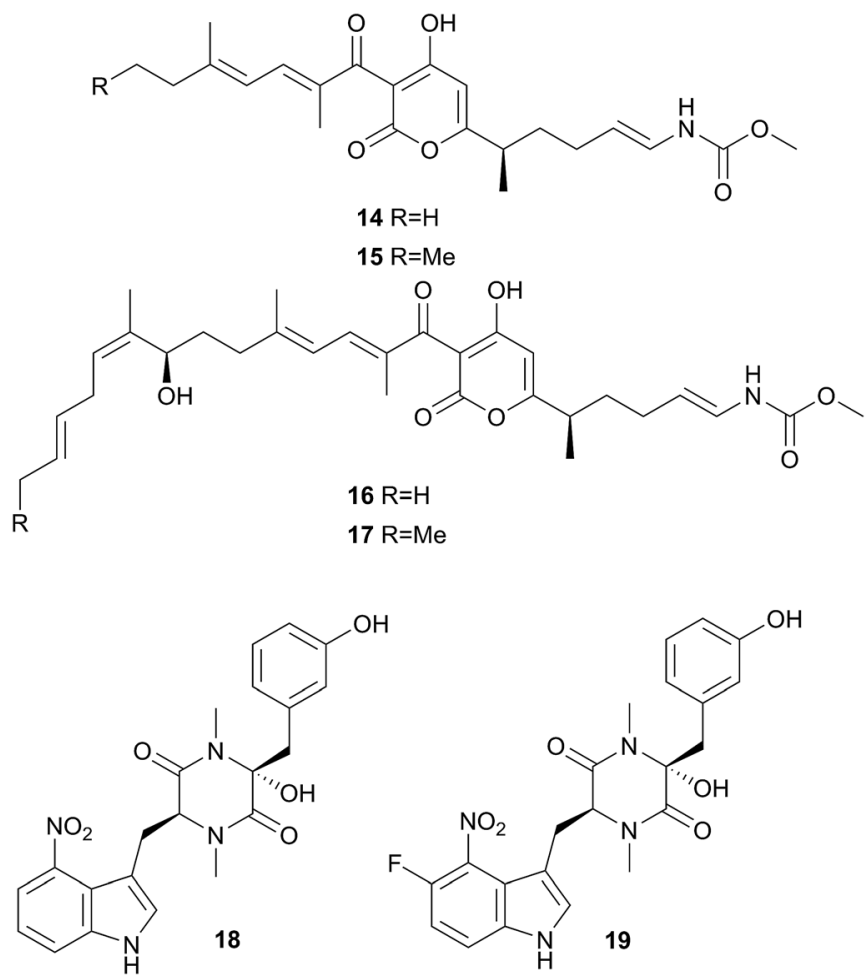

The epothilone BGC including the promoter sequence was randomly integrated into $M$. xanthus. The resulting yields of antitumor active epothilones A $\mathbf{9}$ and B $\mathbf{1 0}$ in the best transposition recombinant ZE9 reached $1.2 \mathrm{mg} \mathrm{L}^{-1}$ in CYE medium. ${ }^{95}$ Inactivation of the esi gene encoding a negative transcription regulator together with medium optimization (CMO medium) significantly stimulated the total yield of epo-

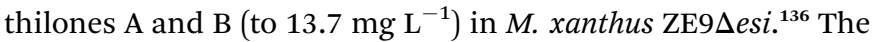
same group developed a CRISPR/mxdCas9-based method using sgRNA to guide the mxdCas9-activator to improve epothilone BGC transcriptional efficiency and consequently increase the production of epothilones in $M$. xanthus, thereby boosting the yields of epothilone A and B from $7 \mathrm{mg} \mathrm{L}^{-1}$ (ZE9) to $25 \mathrm{mg} \mathrm{L}^{-1}$ in CYE medium. ${ }^{\mathbf{1 3 7 , 1 3 8}}$ They also replaced the original epothilone BGC promoter with the strong endogenous promoters $P_{\text {pila }}$ and $P_{\text {groEL1 }}$ in $M$. xanthus; however, epothilone production subsequently declined because the resultant transcriptional activity clearly decreased after the exponential growth stage. This is likely due to the fact that the original promoter, $P_{\text {epo }}$, is predominantly functional in both the late growth stage and the stationary stage and both of these stages are associated with 
optimal epothilone production. Tandem repeat engineering on the original promoter $P_{\text {epo }}$ enhanced the transcriptional level of epothilone genes by approximately twofold and the yields of epothilones were increased from $10 \mathrm{mg} \mathrm{L}^{-1}$ in ZE10 to $21.8 \mathrm{mg} \mathrm{L}^{-1}$ in $\mathrm{ZE} 10-3 \mathrm{CP}_{\text {epo }}$ (containing three copies of the tandem-repeat promoter). ${ }^{139}$ In addition, Bian et al. used the Burkholderiales strain DSM 7029 (Section 3) as an amenable heterologous host to produce epothilones with associated yields reaching $307 \mu \mathrm{g} \mathrm{L}^{-1} \cdot{ }^{109}$ More recently, Liu et al. overexpressed two putative transport genes, open reading frame orf14 and orf3, leading to a twofold increase in the yield of epothilones while simultaneously increasing the ratio of extracellular to intracellular epothilones. ${ }^{\mathbf{1 4 0}}$ These augmentative epothilone production methods could be applied to individual heterologous mutant host strains to further improve associated yields and hopefully generate strains suitable for industrial application in the future.

Thaxtomins (A 18) are virulence factors of common scab potato disease caused by pathogenic Streptomyces strains. They are the main active ingredient of a bioherbicide. ${ }^{\mathbf{1 4 1 - 1 4 3}}$ The transfer of thaxtomin BGC from Streptomyces scabiei into the nonpathogenic $S$. albus $\mathrm{J} 1074$ led to a 10 -fold increase in yield compared to the native producer. The feeding of unnatural precursor 5-fluoro-L-tryptophan in the culture medium resulted in the production of one unnatural fluorinated analog 19 with similarly potent herbicidal activity. ${ }^{\mathbf{1 4 4}}$ Meanwhile, Winn et al. assembled thaxtomin NRPS genes as well as genes encoding a promiscuous tryptophan synthase (TrpS) from Salmonella typhimurium in the heterologous host $S$. albus. Feeding of indole derivatives to the engineered $S$. albus strain enabled the biosynthesis of tryptophan with different substituents, which were then incorporated by the NRPS to generate thaxtomin derivatives with diverse moieties. This work created a de novo biosynthetic pathway to "non-natural" product of thaxtomin in a heterologous host containing multiple enzymes from different pathways and origins. ${ }^{\mathbf{1 4 5}}$

Heterologous expression of BGCs usually led to relatively reduced yields at the initial attempt. However, following medium optimization and host genome engineering strategies (e.g., deletion of large native BGCs and/or BGC manipulations enabling increases in transcriptional and protein expression levels), target production yields often increase significantly. Heterologous expression represents a feasible strategy to improve the yield or titer of desired products from slow-growing sources.

\section{Identification of biosynthetic pathways for known natural products by heterologous expression}

Although numerous studies have given rise to great progress in understanding natural product biosynthetic machinery, a number of intriguing complex structures of natural products still cannot be correlated to the corresponding biosynthetic origins by currently commonly used in silico analysis tools, such as antibiotics and secondary metabolite analysis shell (antiSMASH). ${ }^{146}$ In such cases, heterologous expression is able to find the connection between specific BGCs and particular complex natural products, especially those following a "nontextbook" biosynthetic logic.

The identification of the myxobacterial polyene dawenol 20 biosynthetic pathway revealed a "non-textbook" PKS to be required for this biosynthesis. ${ }^{147}$ Feeding experiments in the wild-type producer Stigmatella aurantiaca DW4/3-1 with isotopically labeled precursors combined with retrobiosynthetic considerations, antiSMASH analysis and different geneinactivation experiments in the wild-type producer led to the identification of the putative dawenol biosynthetic region, which was then used for heterologous expression. Plasmidbased subcloning of the proposed BGC from the genome of $S$. aurantiaca DW4/3-1 was achieved via restriction digestion and Red/ET recombineering was further employed to delete genes not connected to the biosynthesis of dawenol. Mx9 phageintegrase mediated the integration of the expression construct into the chromosome of $M$. xanthus. ${ }^{148}$ Successful isolation of dawenol from the heterologous expression batch confirmed the proposed BGC, which established a system for studying the underlying intriguing biosynthesis. This example showed that heterologous expression provides a practical means to identify the biosynthetic pathways of complex molecules encoded by "non-textbook" BGCs. Combined with bioinformatic analysis, precursor-feeding and gene-inactivation experiments, heterologous expression may become a powerful complementary tool for the identification of biosynthetic genes for structurally known complex natural products in the future.

Vioprolides (A 11) are a promising class of anticancer and antifungal lead compounds produced by the myxobacterium Cystobacter violaceus $\mathrm{Cb}$ vi35. The underlying biosynthesis remained elusive due to the inaccessible genetic manipulation of the native producer. Yan et al. reconstituted the vioprolide BGC by multi-step Red/ET recombination and transferred it into three heterologous hosts, M. xanthus, Burkholderia DSM 7029 and Pseudomonas putida KT2440 for successful expression. ${ }^{110}$ The heterologous expression combined with genetic engineering as well as fermentation optimization not only validated the vioprolide BGC but also improved the yields in an established host, thus supplying sufficient substance for drug development in the future.

PTM compounds are a growing family of natural products with distinct bioactivities. The common biosynthetic origin of PTMs had been identified by genome analyses and genetic manipulation of the native producing organism. ${ }^{149}$ Recently, researchers demonstrated the associated biosynthetic mechanism based on various heterologous expression systems. ${ }^{\mathbf{1 5 0 - 1 5 3}}$ For instance, Gulder and colleagues identified a compact threegene cluster responsible for ikarugamycin $\mathbf{2 1}$ biosynthesis by the first successful heterologous expression of the PTM BGC in E. coli BAP1, ${ }^{150}$ which contains a chromosomal copy of $s f p-$ a gene encoding a promiscuous PPTase - controlled under the T7 promoter. ${ }^{154}$ This was the first successful example of using $E$. coli for heterologous expression of a bacterial iterative PKS system. Although only limited cases show E. coli to be a promising host for heterologous expression of PKS/NRPS compounds, this study established a valuable platform for the 
discovery of novel potent bioactive PTMs. The simplicity of the BGC enabled subsequent bioengineering studies. A similar expression system was successfully employed by the Moore group to identify alterochromide A 22 lipopeptide BGCs from themarine bacterium Pseudoalteromonas piscicida JCM 20779 via heterologous expression in E. coli under the control of both native and T7 promoters. ${ }^{60}$ TAR cloning (refer to the discussion in Section 1) was performed to capture the $34 \mathrm{~kb}$ putative biosynthetic gene cluster. ${ }^{155}$ This successful cloning procedure highlighted the simple and fast exploration of the natural product biosynthetic potential in this promising marine genus Pseudoalteromonas. Recently, the same team used an identical strategy to "capture" and identify the biosynthetic origin of the antitumor agent cosmomycin in Streptomyces coelicolor M512. ${ }^{67}$ Through mass spectral molecular networking, the novel cosmomycin C 23 was also discovered based on the established heterologous system, indicating that the host could be engineered to produce more analogs of the antitumor agent for bioactivity screening in the future.<smiles>C/C=C\C(C)=C/C=C/C(C)=C/C=C/C(C)=C/C(C)/C=C(\C)C(O)C(C)C(C)OC(C)=O</smiles>

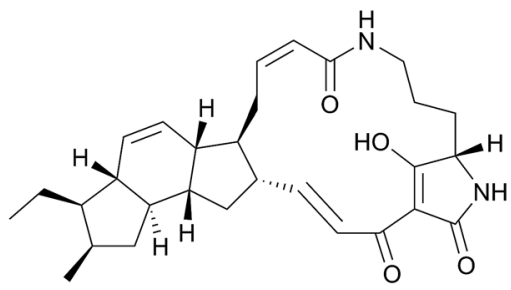

21 spp. $^{156}$ Systemic gene-inactivation in a heterologous host resulted in the identification of the minimum set of genes required for the unique thiopyrano indole 3-methylation. In another independent study, the chuangxinmycin BGC was reconstructed by a DNA assembler from four DNA fragments generated by PCR, but containing a point mutation in one gene, leading to an early termination of translation. ${ }^{157}$ To finally confirm the chuangxinmycin BGC, the mutated gene was complemented by a functional copy of this gene for the successful heterologous expression.

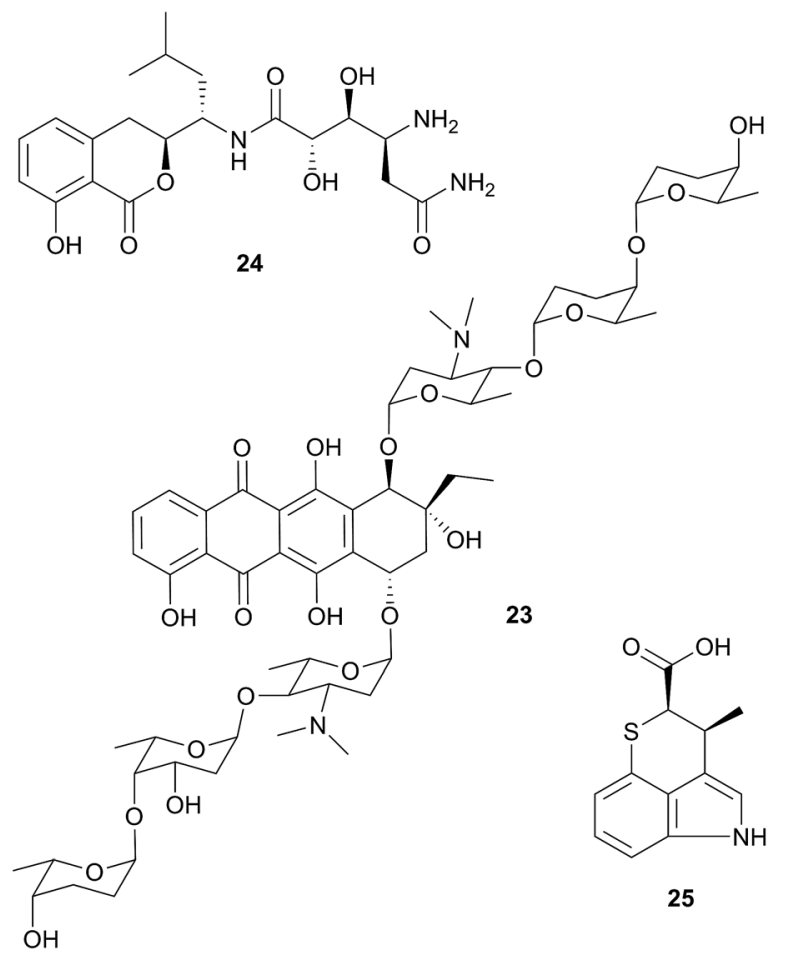

FR900359 26, a potent and selective inhibitor of Gq proteins, was initially isolated from the tropical plant Ardisia crenata. The sequencing of an uncultured endosymbiont, Candidatus Burkholderia crenata, of A. crenata revealed that FR900359 is synthesized by an NRPS in this endosymbiont. The BAC clone containing the FR900359 BGC was modified by insertion of a constitutive promoter and the resultant construct was co-expressed with genes encoding chaperones in E. coli at $16{ }^{\circ} \mathrm{C}$. Although this approach resulted in an extremely low yield $\left(1 \mu \mathrm{g} \mathrm{L} \mathrm{L}^{-1}\right)$, the group was able to confirm the completeness of the FR900359 BGC in the heterologous host and determine its structure. ${ }^{158}$ The fermentative production of FR900359 in a cultivable bacterium following yield optimization would alleviate problems associated with laborious extraction and isolation procedures required in plants, while also facilitating the generation of analogs for pharmacological evaluation by biosynthetic engineering.

Pyridinopyrones were discovered in marine Streptomyces sp. CNQ-301; ${ }^{159}$ however the corresponding BGC was never characterized. Expression of the cryptic iterative type I PKS cluster from S. albus subsp. chlorinus NRRL B-24108 in S. albus Del14 led to the discovery that this cluster encodes the production of pyridinopyrones (A 27). ${ }^{93}$ The activated cryptic pyridinopyrone cluster 
herein encodes an iterative type I PKS synthase consisting of a single module. Primed with nicotinic acid, this module catalyzes six elongation steps to synthesize pyridinopyrones. In the same study, the authors could assign the function of the cryptic type II PKS gene cluster from Frankia sp. CcI3 to be responsible for bhimamycin A and aloesaponarin II biosynthesis. ${ }^{93}$

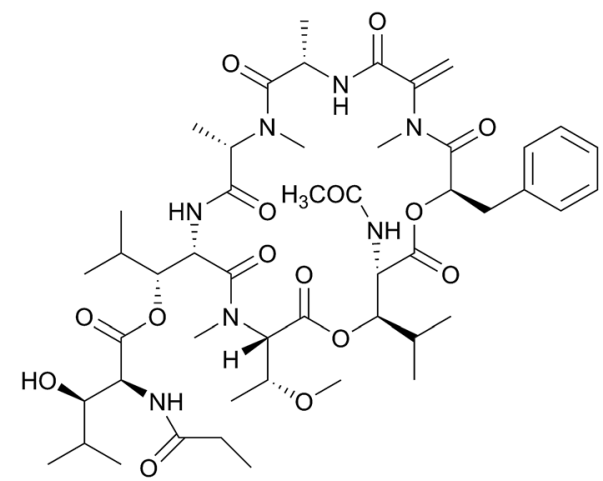<smiles>COc1cc(/C=C/C=C/C(C)=C/C=C/c2cccnc2)oc(=O)c1</smiles>

27

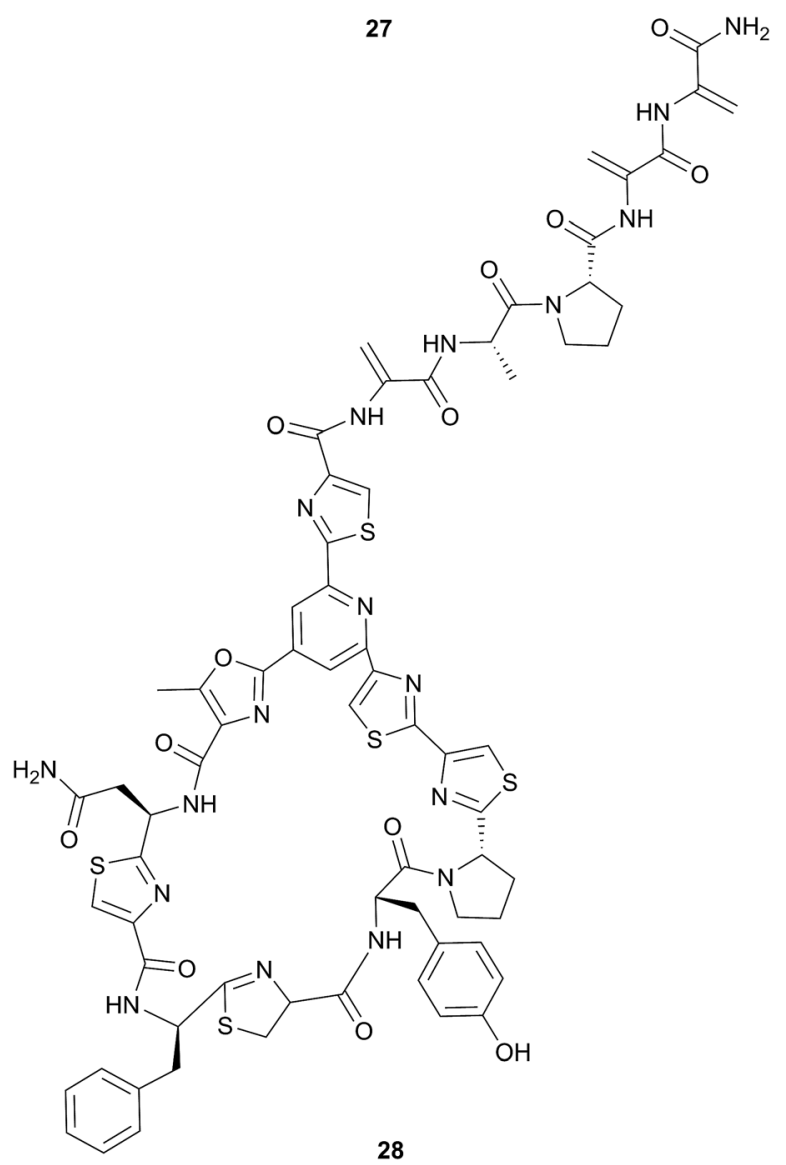

Apart from the heterologous expression of PKS/NRPS systems, the BGCs belonging to RiPPs are mostly easier to predict by in silico analysis and retro-biosynthetic considerations based on the amino acid sequences constituting the core structures and corresponding post-translational modifications. Schäberle and colleagues successfully isolated the antibacterial thiopeptide kocurin from S. coelicolor M1146 under a particular culturing condition..$^{160}$ A putative kocurin 28 BGC spanning nine ORFs and $12 \mathrm{~kb}$ was identified, although the production titer remains low. This might be due to toxicity to the heterologous host, since delayed biomass accumulation was observed. This implies the considerable need for further engineering of the heterologous host, e.g. introducing a self-resistant gene, exchange with an inducible promoter or attempts with other appropriate hosts, in order to obtain a reasonable yield, although the current approach is sufficient for a proof-of-concept study to identify the corresponding gene cluster. The general challenges of heterologous expression of antibiotics in Streptomyces have already been collectively discussed by Apel $e t$ al. ${ }^{161}$

\section{Discovery of novel compounds by heterologous expression of BGCs from cultured bacteria}

The exponentially increasing availability of bacterial genome and metagenome sequences has given rise to enormous biosynthetic potentials for novel natural products. Typically, microbes only express a few BGCs that result in decent yields of respective compounds under laboratory fermentation conditions. The majority of BGCs exhibit very low or even undetectable yields of the respective compounds, severely limiting the screening and discovery of novel natural products, such that the great majority of biosynthetic pathways remain unexploited. Furthermore, although a few techniques are applied to activate cryptic BGCs, an efficient platform is still missing to connect genetic information to biomolecules. Compared with native producer strains, expression in heterologous hosts could be a powerful alternative approach to identify and characterize the unknown biosynthetic pathways using advanced genetic tools.

\subsection{Expression of cryptic BGCs}

A sequence-guided approach has commonly been employed to pre-select the potential BGCs of interest, which relies heavily on bioinformatic analysis based on known biosynthetic logics. Conversely, Tao and colleagues developed a function-guided approach termed a "high-throughput genomic library expression analysis system" (LEXAS) by establishing BAC libraries in Streptomyces spp. to rapidly perform whole-genome screening for bioactive metabolites. They employed BAC vector pHL921 to construct a genomic library of S. rochei Sal35. Each BAC clone was individually transferred into S. lividans SBT5 as the surrogate host, followed by high-throughput antimicrobial activity screening. Along with the two known antibiotics streptothricin and borrelidin, two novel linear lipopeptides (8D1-1 29, 8D1-2 30) and an uncharacterized antibiotic from $S$. rochei Sal35 were identified. ${ }^{162}$ It is noteworthy that although the rediscovery of known bioactive compounds remains an issue, this functional genome mining approach is scalable and adaptable for the industrial screening of 


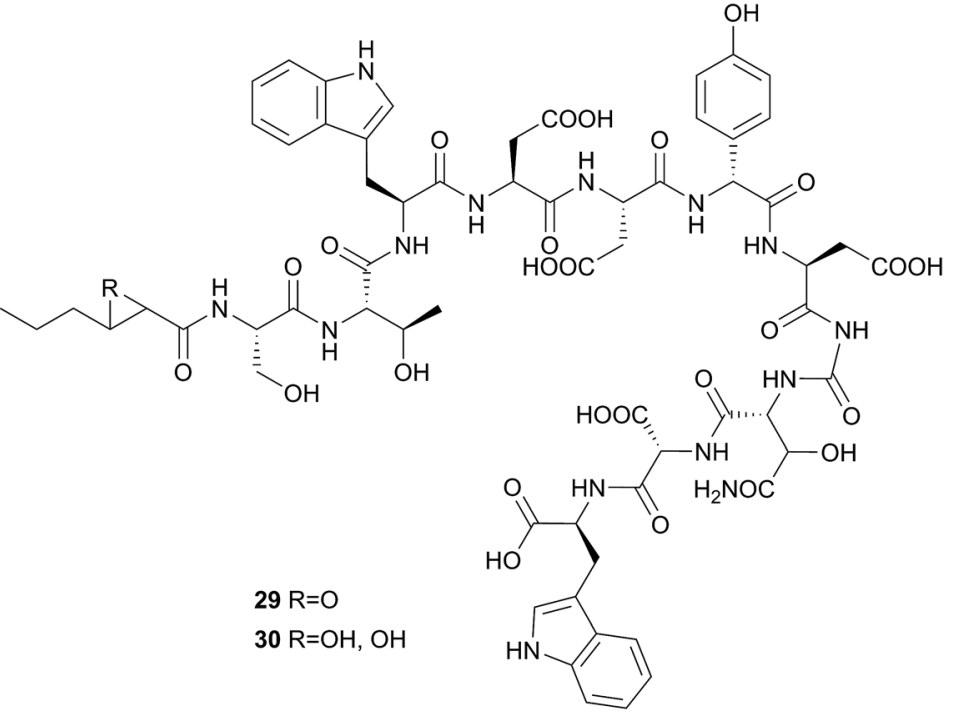

$29 \mathrm{R}=\mathrm{O}$

$30 \mathrm{R}=\mathrm{OH}, \mathrm{OH}$

novel antibiotics in a biotechnological perspective, since the gene mobilization, expression and bioactivity screening can theoretically be performed in a high-throughput fashion.

Advanced genetic manipulation techniques (mentioned in Section 2) facilitate the efficient and precise capture of biosynthetic genes as well as their mobilization into appropriate heterologous hosts. There have been a number of successful examples for BGCs of known function, whereas activation of downregulated BGCs in heterologous systems has resulted in the discovery of few novel natural products and/or derivatives thereof (collective examples are summarized in Table S1†).

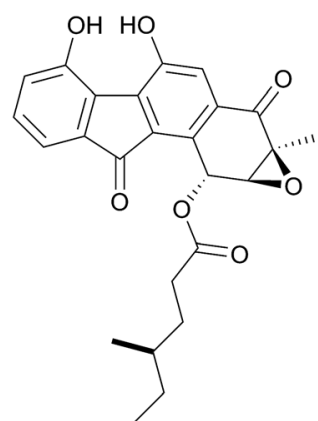

31

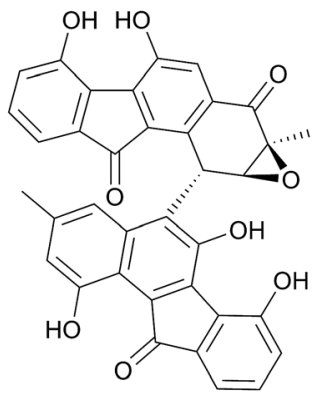

32

Moore and colleagues employed TAR cloning to directly clone and refactor a "silent" NRPS BGC (67 kb) from the marine actinomycete Saccharomonospora sp. CNQ-490, and transfer it into the model heterologous host $S$. coelicolor. Chromosomal integration-based expression revealed two novel lipopeptide antibiotics, taromycin A 2 and B 3, ${ }^{57,58}$ which are structurally similar to the clinically approved antibiotic daptomycin. This study provides a validated approach to efficiently correlate orphan biosynthetic pathways to potentially pharmaceutically relevant metabolites. In addition, two novel marine-derived compounds, fluostatin L $\mathbf{3 1}$ and difluostatin A 32, were identified by Zhang and colleagues. ${ }^{163}$ The putative $40 \mathrm{~kb} f l s$ gene cluster was cloned from marine-derived Micromonospora rosaria SCSIO N160 and successfully expressed in S. coelicolor YF11. Interestingly, production of fluostatins could only be observed

in $S$. coelicolor YF11 when sea salts were supplemented. Difluostatin A exhibited an unusual heterodimer structure and potent antibacterial activity. ${ }^{163}$ Furthermore, Li's group discovered a series of $\alpha$-pyrone compounds named violapyrones from deep sea-derived Streptomyces somaliensis SCSIO ZH66 by heterologously expressing a gene encoding the type III PKS VioA in terrestrial-derived $S$. coelicolor M1146 and marine-derived Streptomyces sanyensis FMA under the control of the constitutive promoter $P_{\text {gapDH }}{ }^{164}$ Five of the 14 isolated violapyrone compounds (VLP2 33, VLP3 34, VLP4 35, VLP7 36, VLP12 37) showed novel structures and one of them exhibited considerable anti-influenza A (H1N1) activity. All these aforementioned studies demonstrated that heterologous expression can serve as a promising tool to uncover novel marine-derived bioactive compounds, which remain largely unexploited due to the difficulties in scalable cultivation and the lack of efficient genetic manipulation tools in the native marine producers.<smiles>Cc1c(O)cc(CCCC(O)C(C)C)oc1=O</smiles>

33

34<smiles>CCc1c(O)cc(CCCCC(C)C)oc1=O</smiles><smiles>CCC(C)(O)CCCCc1cc(O)c(C)c(=O)o1</smiles>

Soon after the PTM biosynthetic pathways had been identified, ${ }^{\mathbf{1 4 9 , 1 5 0}}$ heterologous expression combined with bioengineering efforts (e.g. promoter exchange) enabled the expression 
of previously "silent" PTM pathways. Novel cytotoxic pactamides A-F (A 38) from the marine Streptomyces pactum SCSIO 02999 were discovered in $S$. lividans TK64 by heterologous expression of a PTM BGC under the control of the $\mathrm{ermE}^{*}$ promoter. ${ }^{152}$ Another class of PTM, known as combamides A-D, from Streptomyces sp. S10 were isolated in the heterologous host Streptomyces sp. LZ35derived strain SR111, in which seven native secondary metabolites BGCs were deleted. ${ }^{151}$ The strong constitutive promoter $k a s O^{*}$ was inserted in front of the combamide BGC $(\mathrm{cbm})$, enabling a reasonable production yield $\left(1.5-3 \mathrm{mg} \mathrm{L}^{-1}\right)$. Furthermore, two new derivatives, combamide $\mathrm{E} 39$ and $\mathrm{F}$ 40, containing an additional six-membered ring were generated via co-expression with the OX4 enzyme responsible for the formation of the sixmembered ring in the 5,5,6-tricyclic structure of the heat-stable antifungal factor (HSAFs). ${ }^{151,152,165}$ The Du and Shen groups employed two "clean" Streptomyces hosts, in which the native PKSs have been deleted, to express the HSAF BGCs, leading to the generation of two new HSAF analogs. ${ }^{166}$ In addition, Zhao and colleagues utilized a simple plug-and-play synthetic biology approach to successfully activate a cryptic BGC in Streptomyces griseus. ${ }^{75}$ Six consecutive promoters were inserted in front of each biosynthetic gene, and heterologous expression of the engineered BGC in S. lividans allowed identification of one known compound, alteramide A, and three novel PTMs. For a further overview of recently discovered PTMs via genome mining with a focus on biosynthesis, we refer to the review of Zhang et al. ${ }^{167}$

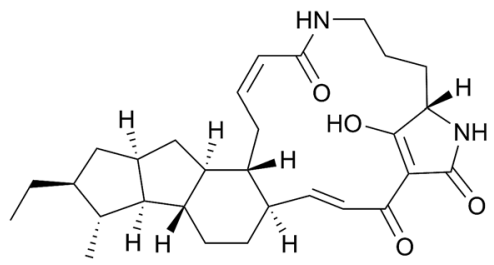

expression since the producer strain $P$. larvae is the causative agent of the fatal honey bee disease American Foulbrood. Thongkongkaew et al. activated an orphan BGC from the plant pathogen Burkholderia glumae PG1 by LLHR cloning and heterologous expression in E. coli GB05-MtaA, leading to the production of two novel lipopeptides, burrioglumin A 42 and B 43, harboring an unusual threonine-tag, which indirectly enabled bacterial colonization in the host via swarming and biofilm formation. ${ }^{168}$

Bode and colleagues employed the ExRec method to reconstruct 15 BGCs from Photorhabdus and Xenorhabdus. A plasmidbased expression in E. coli DH10B-MtaA under the control of T7 promoter allowed the identification of two novel NRPS compounds, ambactin 44 and xenolindicin A 45. ${ }^{76}$ Crawford and colleagues identified two new pteridine metabolites, pepteridine A (46) and B (47), as well as the known 7,8-dihydroxanthopterin and pterin by heterologous expression of a hybrid NRPS-pteridine genomic island from the entomopathogen Photorhabdus luminescens TT01 in E. coli BAP1 under the control of a phage T7 promoter. ${ }^{169}$ In addition, Nihira et al. discovered a novel diol-containing PKS lavendiol 48 from Streptomyces lavendulae by expressing the unique BGC of such streptenolfamily compounds. ${ }^{170}$ This established the heterologous platform for the rational design of novel lavendiol derivatives for further bioactivity screening. Gulder and colleagues employed DiPaC to capture the "silent" ehp gene cluster from Serratia fonticola DSM 4576, followed by the heterologous expression in E. coli BAP1, yielding a novel phenazine derivative fontizine A 5. ${ }^{77}$ Cai et al. discovered six new nevaltophins A-F (A 49) derived from Xenorhabdus PB62.4 by expression of $p b 62 A$ and $p b 62 B$ BGCs in E. coli. ${ }^{171}$ Nevaltophins possess an additional L-valine moiety and tryptamine or phenylethylamine as the terminal amine compared with the known antibiotic nematophin, ${ }^{171}$ which contributed to the structure diversity of bioactive compounds from entomopathogenic bacteria.

Heterologous expression of cryptic RiPP BGCs was exemplified by the successful isolation of the cytotoxic neothioviridamide $\mathbf{5 0}$ from $S$. avermitilis SUKA22, a new polythioamide compound originating from Streptomyces sp. MSB090213SC12. ${ }^{172}$ This heterologous expression system set the stage for diversifying RiPP structures by simple exchange of amino acid residues in the core peptide sequences. However, the promiscuity of the tailoring enzymes must be taken into account when the precursor peptide sequence changes. This issue has been comprehensively reviewed by Funk and van der Donk. ${ }^{13}$

\subsection{Biosynthetic engineering/combinatorial biosynthesis}

Improved genetic tools such as Red/ET recombineering can not only directly clone large BGCs, facilitating the horizontal gene transfer into the heterologous host, but also provide an efficient means for the seamless modification of the biosynthetic genes as well as corresponding regulatory elements. ${ }^{173}$ Much genetic engineering of biosynthetic pathways in heterologous hosts has been successfully conducted with the aim of generation of novel analogs and yield optimization as well as an in-depth understanding of biosynthetic mechanisms. These studies include the feeding of biosynthetic precursors, promoter engineering,
Based on the LLHR strategy, a $11.6 \mathrm{~kb}$ cryptic NRPS BGC from Paenibacillus larvae was successfully cloned and heterologously expressed in E. coli GB05-MtaA, resulting in the production of a novel compound, sevadicin $\mathbf{4 1 .} .^{40}$ Despite the fact that no antimicrobial activity was detected, it is suggested that the compound may play a role in pathogenesis as a regulator of virulence factor 
<smiles>C/C=C(\CC(=O)N[C@@H](O)CCCCCCC)C(=O)N1CCC[C@H]1C(=O)N[C@@H](CO)C(=O)N[C@@H](C)C(=O)N[C@@H](C(=O)N[C@@H](Cc1ccccc1)C(=O)N1CCC[C@H]1C(=O)N[C@@H](C(=O)O)[C@@H](C)O)C(C)C</smiles>

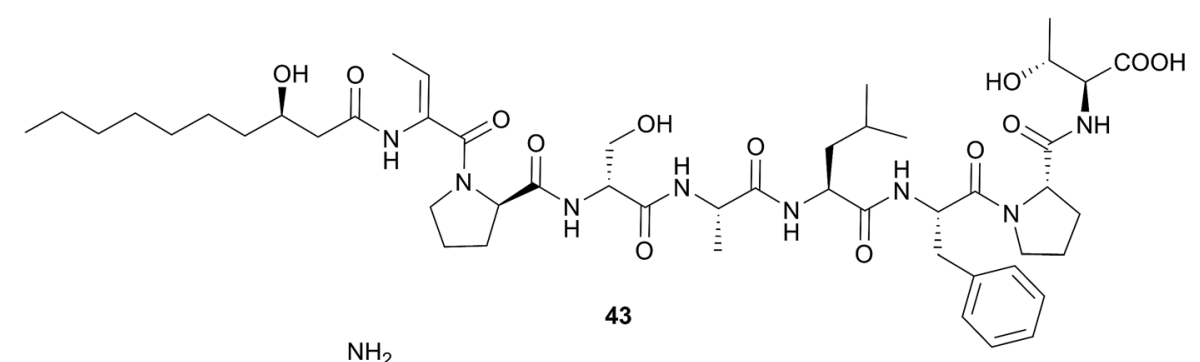

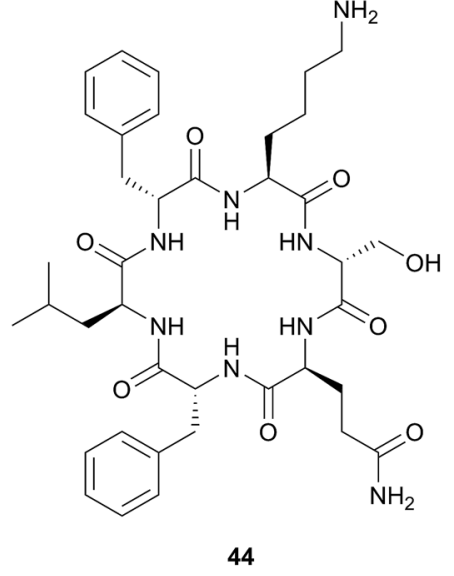<smiles>C/C=C/C=C/C=C/C(=O)C(C)[C@H](O)CCO</smiles>

48<smiles>CCC(C)C(=O)C(=O)N(C)C(C(=O)NCCc1ccccc1)C(C)C</smiles>

49<smiles>CSC=CNC(=O)C(NC(=O)C(Cc1ccccc1)NC(=O)C(C)NC(=O)C(NC(=O)C(NC(=O)C(C)NC(=S)C(C)NC(=O)C(C)NC(=O)C(C)NC(=S)C(CCSC)NC(=S)C(NC(=O)C(C)=O)C(C)C)C(C)SC)C(C)C)C(O)c1c[n+](C)cn1C</smiles> 
manipulation of transcriptional regulators, insertion/deletion of tailoring enzymes, change in copy numbers of BGC transcripts, localization of BGCs on the host chromosomes, or combined approaches thereof. In this sub-section, we discuss only some representative cases, where novel natural product analogs have been obtained by these bioengineering approaches (for a collective summary of the compounds, see Table S1 $\dagger$ ).

Bode and colleagues generated a great number of novel rhabdopeptide/xenortide peptides (RXPs) originating from entomopathogenic bacteria Photorhabdus and Xenorhabdus in E. coli. ${ }^{174}$ Diverse peptides (51-53) are produced in nature due to the flexible and iterative utilization of NRPS biosynthetic enzymes. ${ }^{175}$ The authors employed biosynthetic engineering approaches, including domain swaps, module skipping and cross-talk, to alter the biosynthetic pathways, enabling the identification of even more bioactive RXP derivatives. ${ }^{174}$ The same team recently developed a new strategy for the modification of NRPSs, where exchange units (XUs) rather than modules are defined as functional units. By obeying the specificity of the native downstream condensation (C) domain, fusion of XUs at specific positions enabled the production of desired new peptides in great diversity. Release of the mature peptide from the NRPS is conventionally catalyzed by a thioesterase (TE) domain or a terminal C domain by hydrolysis or cyclization via intramolecular nucleophiles. ${ }^{176}$ Interestingly, the authors were able to employ the internal $\mathrm{C}$ domains to release the peptide chains, yielding various cyclic peptides, ${ }^{175}$ a strategy which could circumvent the limited specificity of TE domains. The XU concept set up a new platform for the production of specific non-ribosomal peptides in a scalable process.

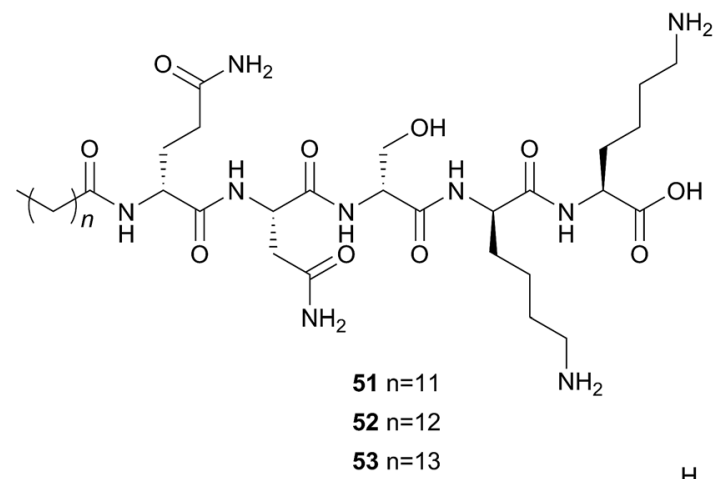

Based on myxochromide BGCs, Wenzel, Müller, and colleagues used synthetic biology approaches to explore the diversity of this type of lipopeptide. By combinatorial exchange of NRPS genes, a number of artificial myxochromide BGCs have been made to successfully produce novel hybrid myxochromides $\left(\mathrm{AS}_{4}, \mathbf{5 4}\right)$ in a derivative of Myxococcus xanthus DK1622. ${ }^{177}$ This newly developed assembly strategy of complex synthetic BGCs based on type IIS endonucleases offers great potential to accelerate the process of biosynthetic pathway engineering.

$\mathrm{Lu}$ and colleagues also demonstrated a combinatorial biosynthesis example to obtain a series of novel unnatural nonribosomal siderophores in $E$. coli by building a compressed biosynthetic gene cassette combined with supplementation of various polyamine precursors and corresponding pressuring culture conditions. ${ }^{178}$ Blodgett and colleagues discovered a novel cytochrome P450 enzyme CftA in Streptomyces sp. JV178 and performed the heterologous expression of $c f t A$ isozyme genes in the known ikarugamycin producer, Streptomyces sp. NRRL F-2890, successfully yielding a new PTM, the ketone-containing clifednamide A 55. ${ }^{153}$ Luzhetskyy and colleagues derivatized a ribosomally synthesized antibiotic cinnamycin via incorporation of non-natural amino acids in heterologous host $S$. albus. ${ }^{179}$ This opens a way to incorporate new functional groups into RiPPs using heterologous expression of corresponding BGCs.

It is noteworthy that limitations pertaining to the recombineering of repeat sequences could occasionally restrain the application of Red/ET technology, since repeated sequences frequently exist in most PKS/NRPS biosynthetic genes. Traditional cloning methods or other DNA engineering techniques, such as Gibson assembly or CRISPR/Cas, might alternatively be used in such situations.

\section{Discovery of novel compounds by heterologous expression of DNA from uncultured bacteria and metagenomic DNA}

As little as $1 \%$ of bacteria from the environment have been cultured and certainly less than this have been exploited for natural product production. ${ }^{180}$ An increasing number of sequencing projects have revealed the enormous biosynthetic potential of unexplored natural products by environmental microbiomes. Functional metagenomics has realized the investigation of natural product biosynthetic pathways from uncultured bacteria. ${ }^{181}$ Efficient mobilization of such metagenomic DNA into a cultured bacterium and subsequent heterologous expression in a surrogate host provide the chance to comprehensively correlate environmental DNA samples and their encoded secondary metabolites.

Brady and colleagues pioneered the discovery of a number of novel bioactive natural products through metagenomic library screening and subsequent heterologous expression of soil environmental DNA-derived genes in various hosts. ${ }^{\mathbf{1 8 2 - 1 8 8}}$ For instance, methylarcyriarubin $\mathbf{5 6}$ was isolated from $E$. coli after expression of the putative mar BGC with an exogenous oxidized tryptophan (IPA imine) synthase encoding gene. This represented the first report of methylarcyriarubin being isolated as 
<smiles>CN1C(=O)C(c2c[nH]c3ccccc23)=C(c2c[nH]c3ccccc23)C1=O</smiles><smiles>O=C1NCc2c1c1c3cc(O)ccc3[nH]c1c1[nH]c3ccc(O)cc3c21</smiles>

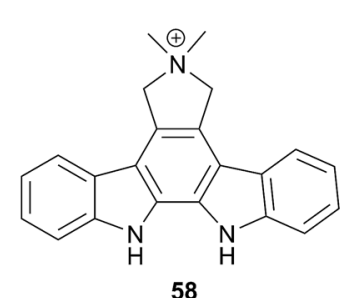

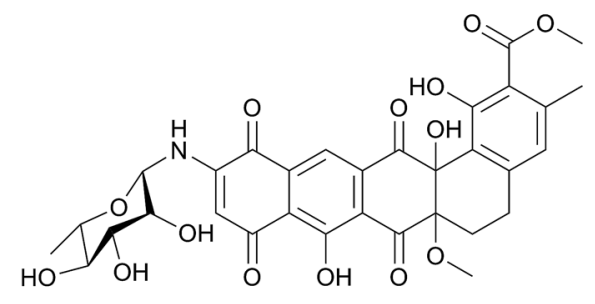

61

63 a natural product. ${ }^{182}$ To overcome limitations in relation to the screening of current eDNA libraries, the same team performed a chemical-biogeographic survey to assess chromopyrrolic acid synthase (CPAS) gene diversity, resulting in the identification of two bioactive tryptophan dimers (TDs): hydroxysporine 57 and reductasporine 58. The latter molecule contains a previously unidentified pyrrolinium indolocarbazole core, which might be correlated to its unusual bioactivity. ${ }^{183}$ In addition, PCR-based screening approaches amplifying $\mathrm{KS}_{\beta}$ domain-encoding sequences of type II PKS facilitated the identification of the corresponding BGCs from an eDNA library. TAR-based assembly was employed for the recovery of entire BGCs from a metagenomic library followed by integration into the genome of $S$. albus by bacterial phage phic31. Three novel bioactive pentangular polyphenols, arixanthomycin A 59 exhibiting potent antiproliferative activity against human cancer cell lines and arenimycins $\mathrm{C} 60$ and D 61 displaying potent antibacterial activity, were isolated from $S$. albus. ${ }^{187}$ A similar approach was used to identify arimetamycin A 62 from an eDNA-derived BGC, which exhibited more potent anticancer activity than current clinically used anthracyclines. ${ }^{186}$ All these results represented a well-established platform to express type II PKSs from metagenomics, based on a sufficient supply of the ubiquitous precursors (e.g. malonyl-CoA) and relatively smallsized BGCs. This approach will require further optimisation to functionally express more types of BGCs in multiple hosts.

Seven new epoxyketone proteasome inhibitor (EPI) congeners (clarepoxcins A-E (A 63) and landepoxcin A 64 and B) were isolated from $S$. albus $\mathrm{J} 1074$ through identification and recovery

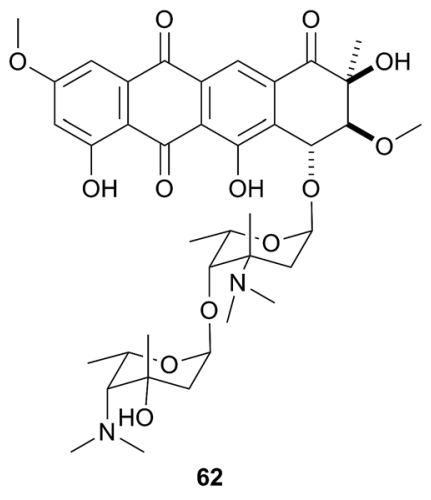<smiles>CC(C)C[C@H](C(=O)N[C@H](C(=O)N[C@H](C(=O)N[C@@H](C)C(=O)C1(C)CO1)[C@H](C)O)C(C)C)N(C)C(=O)CCCCCCC(C)(C)O</smiles><smiles>C=C(C)C[C@H](NC(=O)[C@@H](NC(=O)CC(C)C)C(C)(C)C)C(=O)C1CO1</smiles><smiles>NC(=O)/C=C/C=C/C1C=CC2C=CC3OC(/C=C/C=C/C=C/C(=O)O)C1C23</smiles> 
<smiles>CC(C)[C@H](NC(=O)[C@@H](NC(=O)CNC(=O)[C@H](CC(=O)O)NC(=O)[C@@H](C)C(O)C(=O)O)C(C)C(=O)O)C(=O)N1C[C@H](C)C[C@H]1C(N)=O</smiles><smiles>[R]C(C)CC/C=C\C=C\C(=O)N[C@H](C(=O)N[C@H](C(=O)NC(C(=O)N[C@@H](CCCCN)C(=O)N[CH])C(C)C)C(C)N)C(C)C(=O)O</smiles>

$66 \mathrm{R}=\mathrm{Me}$<smiles></smiles>

$67 \mathrm{R}=\mathrm{Et}$

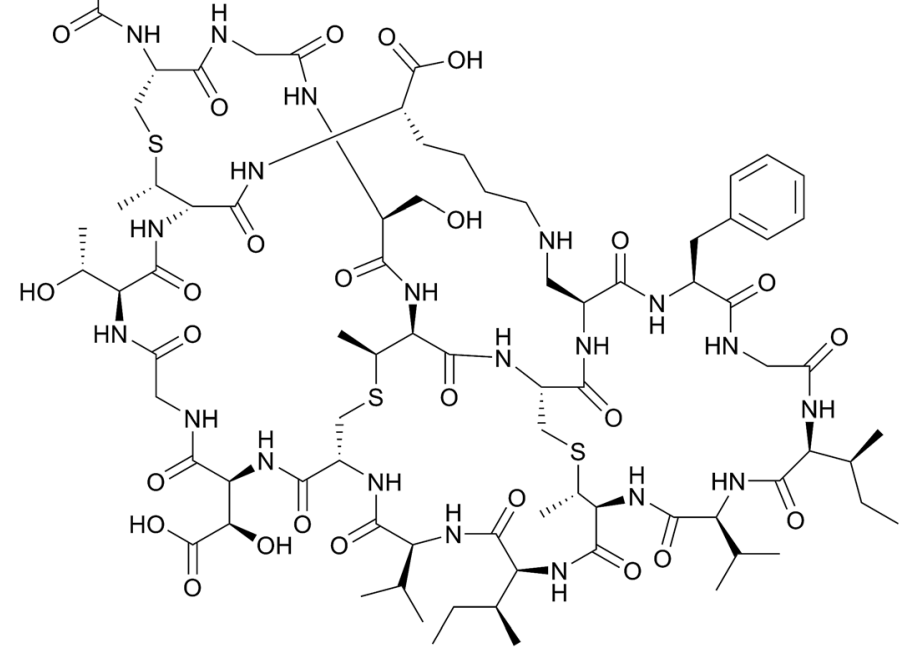

68<smiles>CCC(C)CCCCCCCCCCC(=O)OCC(O)COP(=O)(O)OCCNC</smiles><smiles>CCC(C)C=CC(=O)OC(CC(O)C(C)C=CC=CC(C)CC=C(C)C(O)C=CCC(O)C(C)C=CC(=O)O)C(C)CC(C)C=CC=C(C)C=CC(O)C(C)CC</smiles> 
of the EPI BGCs from four saturating soil metagenomic libraries by using 99 unique epoxyketone sequence tags. ${ }^{188}$ The authors subsequently isolated a novel polyketide natural product, metatricycloene 65, by performing an improved functional metagenomics study in E. coli. ${ }^{\mathbf{1 8 5}}$ Most recently, based on the established sequence-guided metagenomic discovery pipeline, the same group discovered malacidins (A 66, B 67) as a novel class of antibiotics. These antibiotics are active against multidrug-resistant pathogens, including methicillin-resistant Staphylococcus aureus. ${ }^{\mathbf{1 8 4}}$ This study revealed that calciumdependent antibiotics are more diverse than previously thought, while also demonstrating that soil metagenomics could be used to discover novel antibiotics in the future. Interestingly, Nasrin et al. identified three new derivatives of the known antibiotic chloramphenicol, which was used as a selection marker in a functional metagenomic screening process, ${ }^{\mathbf{1 8 9}}$ indicating that this approach could lead to further derivatives of known scaffolds through modification by enzymes rather than obtaining truly novel compounds from novel BGCs from metagenomic libraries. Thus, the issue of identifying truly novel and complex chemical scaffolds from metagenomic DNA remains challenging.

With respect to marine-derived metagenomic advances, Moore and colleagues discovered polybrominated diphenyl ethers (PBDEs) from sponge-microbiome-associated cyanobacterial endosymbionts by heterologously expressing PBDE BGCs in cyanobacterial hosts. ${ }^{190}$ They employed the de novo metagenomic sequencing and assembly approach to perform an amino acid-based rather than a nucleotide-based sequence search. This study led to the identification of putative PBDE gene clusters. Genome integration-based expression of these gene clusters in Synechococcus elongatus PCC 7942 under the control of a synthetic promoter-riboswitch system yielded various PBDEs. ${ }^{190}$ The study resulted in the establishment of a platform for the metagenomic-based exploration of further PBDEs in the marine environment. Smith et al. discovered a novel potent anti-HIV lanthipeptide divamide A 68 from uncultured symbiotic bacteria living in tunicates using heterologous expression of the synthetic BGC in E. coli. ${ }^{191}$ This example revealed that heterologous expression is a powerful tool to obtain sufficient quantities of substances from a very limited resource, allowing the elucidation of the structures of natural products as well as an evaluation of their biological activities. This methodology could also be applied to the investigation of bioactive compounds from complex symbiotic systems.

Fischbach and colleagues employed bioinformatics, synthetic biology, and heterologous expression approaches to discover a series of novel pyrazinones and dihydropyrazinones from human gut microbiota. ${ }^{192}$ In addition, 47 putative NRPS BGCs have been identified by in silico analysis. Based on sequence diversity and domain architectures from various clades, 14 representative clusters were selected for heterologous expression in E. coli and B. subtilis. Successful heterologous expression yielded a number of pyrazinones and dihydropyrazinones. Further investigation demonstrated that these gut NRPS clusters are actively transcribed under conditions of host colonization. More interestingly, active gut NRPS products may initially be released as dipeptide aldehydes, which could serve as potent protease inhibitors. This study provided a platform for the systematic investigation of metabolic profiles associated with human microbiota without the requirement to culture individual strains. This in-depth mining approach might help to realize the biosynthetic potential of human microbiota, thereby making studies on small molecules regulating microbe-microbe and microbe-human interactions more accessible.

Recent environmental microbiome studies by Piel and Vorholt et al. demonstrated that the Arabidopsis leaf microbiome exhibited a promising biosynthetic potential for bioactive natural products. ${ }^{193}$ Here, microbial culturing and coculturing technologies revealed the isolation of various novel antibiotic agents, e.g. phosphobrevin (69) and macrobrevin (70). It can be anticipated that heterologous expression will in the future facilitate the exploration of uncharacterized natural products from phyllosphere and from other microbiomes.

\section{Conclusion}

The discovery of novel antibiotics to combat emerging resistance as well as the identification of new drug leads to treat for example infectious diseases are becoming increasingly desirable nowadays. Natural products will definitely continue to play an irreplaceable role in this field. With the progress of genomics and synthetic biotechnology, heterologous expression of BGCs has been used extensively as an approach to access natural products from bacteria and eDNA samples. In the past five years, heterologous expression has not only enabled the characterization of roughly 70 natural product BGCs from intractable bacteria or metagenomics, but has also provided an efficient means to uncover the products of approximately 30 cryptic BGCs to obtain potentially bioactive novel compounds (Table S1†). Furthermore, heterologous expression also sets the stage for the yield optimization of valuable chemicals of pharmaceutical, agrochemical or nutritional interest. Advanced biotechnology-mediated identification, reconstitution, mobilization, and modification of BGCs will be increasingly employed for further awakening of cryptic BGCs as well as for excavating the biosynthetic potential from eDNAs. Without doubt, it can foreseen that heterologous expression systems combined with synthetic biotechnology will substantially enhance the in-depth understanding of non-textbook biosynthetic mechanisms, the discovery of novel scaffolds from diverse natural resources, and even the rational design and engineering for the artificial generation of novel molecules in the future.

\section{Conflicts of interest}

There are no conflicts to declare.

\section{Acknowledgements}

The authors thank Prof. A. Luzhetskyy (Saarland University) for constructive suggestions for this manuscript and Dr V. 
Ravichandran (SDU) for proofreading of the manuscript. We thank the National Natural Science Foundation of China (31670098, 31670097), the Higher Education Discipline Innovation Introduction Program (B16030), the Recruitment Program of Global Experts (Y. Z.) and the Qilu Youth Scholar Startup Funding of Shandong University (X. B. and L. H.) for financial support. Research in the laboratory of RM was funded by the DFG, the PhD fellowship of the Boehringer Ingelheim Fonds, BMBF and Deutsches Zentrum für Infektionsforschung Standort Hannover-Braunschweig.

\section{References}

1 F. E. Koehn and G. T. Carter, Nat. Rev. Drug Discovery, 2005, 4, 206-220.

2 G. M. Cragg and D. J. Newman, Biochim. Biophys. Acta, 2013, 1830, 3670-3695.

3 D. J. Newman and G. M. Cragg, J. Nat. Prod., 2016, 79, 629661.

4 M. A. Fischbach and C. T. Walsh, Science, 2009, 325, 10891093.

5 A. L. Demain, J. Ind. Microbiol. Biotechnol., 2006, 33, 486495.

6 M. A. Marahiel and L. O. Essen, Methods Enzymol., 2009, 458, 337-351.

7 C. T. Walsh, Science, 2004, 303, 1805-1810.

8 K. J. Weissman, Methods Enzymol., 2009, 459, 3-16.

9 C. Hertweck, Angew. Chem., Int. Ed. Engl., 2009, 48, 46884716.

10 T. Stein, J. Vater, V. Kruft, A. Otto, B. Wittmann-Liebold, P. Franke, M. Panico, R. McDowell and H. R. Morris, J. Biol. Chem., 1996, 271, 15428-15435.

11 M. A. Fischbach and C. T. Walsh, Chem. Rev., 2006, 106, 3468-3496.

12 J. N. Copp and B. A. Neilan, Appl. Environ. Microbiol., 2006, 72, 2298-2305.

13 M. A. Funk and W. A. van der Donk, Acc. Chem. Res., 2017, 50, 1577-1586.

14 K. J. Hetrick and W. A. van der Donk, Curr. Opin. Chem. Biol., 2017, 38, 36-44.

15 P. G. Arnison, M. J. Bibb, G. Bierbaum, A. A. Bowers, T. S. Bugni, G. Bulaj, J. A. Camarero, D. J. Campopiano, G. L. Challis, J. Clardy, P. D. Cotter, D. J. Craik, M. Dawson, E. Dittmann, S. Donadio, P. C. Dorrestein, K. D. Entian, M. A. Fischbach, J. S. Garavelli, U. Goransson, C. W. Gruber, D. H. Haft, T. K. Hemscheidt, C. Hertweck, C. Hill, A. R. Horswill, M. Jaspars, W. L. Kelly, J. P. Klinman, O. P. Kuipers, A. J. Link, W. Liu, M. A. Marahiel, D. A. Mitchell, G. N. Moll, B. S. Moore, R. Müller, S. K. Nair, I. F. Nes, G. E. Norris, B. M. Olivera, H. Onaka, M. L. Patchett, J. Piel, M. J. Reaney, S. Rebuffat, R. P. Ross, H. G. Sahl, E. W. Schmidt, M. E. Selsted, K. Severinov, B. Shen, K. Sivonen, L. Smith, T. Stein, R. D. Süssmuth, J. R. Tagg, G. L. Tang, A. W. Truman, J. C. Vederas, C. T. Walsh, J. D. Walton, S. C. Wenzel, J. M. Willey and W. A. van der Donk, Nat. Prod. Rep., 2013, 30, 108-160.
16 L. Huo, S. Rachid, M. Stadler, S. C. Wenzel and R. Müller, Chem. Biol., 2012, 19, 1278-1287.

17 T. Inaoka, K. Takahashi, M. Ohnishi-Kameyama, M. Yoshida and K. Ochi, J. Biol. Chem., 2003, 278, 21692176.

18 J. Shima, A. Hesketh, S. Okamoto, S. Kawamoto and K. Ochi, J. Bacteriol., 1996, 178, 7276-7284.

19 K. Buntin, H. Irschik, K. J. Weissman, E. Luxenburger, H. Blocker and R. Müller, Chem. Biol., 2010, 17, 342-356.

20 N. S. Cortina, D. Krug, A. Plaza, O. Revermann and R. Müller, Angew. Chem., Int. Ed. Engl., 2012, 51, 811-816.

21 S. Bergmann, A. N. Funk, K. Scherlach, V. Schroeckh, E. Shelest, U. Horn, C. Hertweck and A. A. Brakhage, Appl. Environ. Microbiol., 2010, 76, 8143-8149.

22 K. Sakai, H. Kinoshita and T. Nihira, Appl. Microbiol. Biotechnol., 2012, 93, 2011-2022.

23 S. Rachid, K. Gerth, I. Kochems and R. Müller, Mol. Microbiol., 2007, 63, 1783-1796.

24 A. Sandmann, B. Frank and R. Müller, J. Biotechnol., 2008, 135, 255-261.

25 S. Rachid, K. Gerth and R. Müller, J. Biotechnol., 2009, 140, 135-142.

26 D. N. Mao, L. B. Bushin, K. Moon, Y. H. Wu and M. R. Seyedsayamdost, Proc. Natl. Acad. Sci. U. S. A., 2017, 114, E2920-E2928.

27 S. E. Ongley, X. Bian, B. A. Neilan and R. Müller, Nat. Prod. Rep., 2013, 30, 1121-1138.

28 A. C. Jones, B. Gust, A. Kulik, L. Heide, M. J. Buttner and M. J. Bibb, PLoS One, 2013, 8, e69319.

29 J. Tu, S. Li, J. Chen, Y. Song, S. Fu, J. Ju and Q. Li, Microb. Cell Fact., 2018, 17, 28.

30 B. Kepplinger, S. Morton-Laing, K. H. Seistrup, E. C. L. Marrs, A. P. Hopkins, J. D. Perry, H. Strahl, M. J. Hall, J. Errington and N. E. E. Allenby, ACS Chem. Biol., 2018, 13, 207-214.

31 J. F. Castro, V. Razmilic, J. P. Gomez-Escribano, B. Andrews, J. A. Asenjo and M. J. Bibb, Appl. Environ. Microbiol., 2015, 81, 5820-5831.

32 H. Liu, H. Jiang, B. Haltli, K. Kulowski, E. Muszynska, X. Feng, M. Summers, M. Young, E. Graziani, F. Koehn, G. T. Carter and M. He, J. Nat. Prod., 2009, 72, 389-395.

33 H. J. Nah, M. W. Woo, S. S. Choi and E. S. Kim, Microb. Cell Fact., 2015, 14, 140.

34 H. R. Pyeon, H. J. Nah, S. H. Kang, S. S. Choi and E. S. Kim, Microb. Cell Fact., 2017, 16, 96.

35 Y. Zhou, A. C. Murphy, M. Samborskyy, P. Prediger, L. C. Dias and P. F. Leadlay, Chem. Biol., 2015, 22, 745-754.

36 H. Li, J. Qu, T. Li, S. Wirth, Y. Zhang, X. Zhao and X. Li, Appl. Microbiol. Biotechnol., 2018, 102, 6739-6751.

37 X. Bian, F. Huang, F. A. Stewart, L. Xia, Y. Zhang and R. Müller, ChemBioChem, 2012, 13, 1946-1952.

38 J. Fu, X. Bian, S. Hu, H. Wang, F. Huang, P. M. Seibert, A. Plaza, L. Xia, R. Müller, A. F. Stewart and Y. Zhang, Nat. Biotechnol., 2012, 30, 440-446.

39 X. Bian, F. Huang, H. Wang, T. Klefisch, R. Müller and Y. Zhang, ChemBioChem, 2014, 15, 2221-2224. 
40 Y. Tang, S. Frewert, K. Harmrolfs, J. Herrmann, L. Karmann, U. Kazmaier, L. Xia, Y. Zhang and R. Müller, J. Biotechnol., 2015, 194, 112-114.

41 X. Bian, A. Plaza, Y. Zhang and R. Müller, J. Nat. Prod., 2012, 75, 1652-1655.

42 X. Bian, J. Fu, A. Plaza, J. Herrmann, D. Pistorious, A. F. Stewart, Y. Zhang and R. Müller, ChemBioChem, 2013, 14, 1194-1197.

43 Q. Liu, Q. Shen, X. Bian, H. Chen, J. Fu, H. Wang, P. Lei, Z. Guo, W. Chen, D. Li and Y. Zhang, Sci. Rep., 2016, 6, 34623.

44 H. Wang, Z. Li, R. Jia, Y. Hou, J. Yin, X. Bian, A. Li, R. Müller, A. F. Stewart, J. Fu and Y. Zhang, Nat. Protoc., 2016, 11, 1175-1190.

45 F. Huang, J. Tang, L. He, X. Ding, S. Huang, Y. Zhang, Y. Sun and L. Xia, Microb. Cell Fact., 2018, 17, 31.

46 J. Yin, M. Hoffmann, X. Bian, Q. Tu, F. Yan, L. Xia, X. Ding, A. F. Stewart, R. Müller, J. Fu and Y. Zhang, Sci. Rep., 2015, 5, 15081.

47 H. Wang, Z. Li, R. Jia, J. Yin, A. Li, L. Xia, Y. Yin, R. Müller, J. Fu, A. F. Stewart and Y. Zhang, Nucleic Acids Res., 2018, 46, e28.

48 D. G. Gibson, L. Young, R. Y. Chuang, J. C. Venter, C. A. Hutchison and H. O. Smith, Nat. Methods, 2009, 6, 343-U341.

49 W. Jiang, X. Zhao, T. Gabrieli, C. Lou, Y. Ebenstein and T. F. Zhu, Nat. Commun., 2015, 6, 8101.

50 W. Tang, Z. Guo, Z. Cao, M. Wang, P. Li, X. Meng, X. Zhao, Z. Xie, W. Wang, A. Zhou, C. Lou and Y. Chen, Proc. Natl. Acad. Sci. U. S. A., 2018, 115, 2818-2823.

51 V. Larionov, N. Kouprina, J. Graves, X. N. Chen, J. R. Korenberg and M. A. Resnick, Proc. Natl. Acad. Sci. U. S. A., 1996, 93, 491-496.

52 N. Kouprina and V. Larionov, Nat. Rev. Genet., 2006, 7, 805812.

53 N. Kouprina and V. Larionov, Nat. Protoc., 2008, 3, 371-377.

54 J. H. Kim, Z. Feng, J. D. Bauer, D. Kallifidas, P. Y. Calle and S. F. Brady, Biopolymers, 2010, 93, 833-844.

55 Z. Feng, D. Kallifidas and S. F. Brady, Proc. Natl. Acad. Sci. U. S. A., 2011, 108, 12629-12634.

56 Z. Feng, J. H. Kim and S. F. Brady, J. Am. Chem. Soc., 2010, 132, 11902-11903.

57 K. Yamanaka, K. A. Reynolds, R. D. Kersten, K. S. Ryan, D. J. Gonzalez, V. Nizet, P. C. Dorrestein and B. S. Moore, Proc. Natl. Acad. Sci. U. S. A., 2014, 111, 1957-1962.

58 K. A. Reynolds, H. Luhavaya, J. Li, S. Dahesh, V. Nizet, K. Yamanaka and B. S. Moore, J. Antibiot., 2018, 71, 333338.

59 B. Bonet, R. Teufel, M. Crusemann, N. Ziemert and B. S. Moore, J. Nat. Prod., 2015, 78, 539-542.

60 A. C. Ross, L. E. Gulland, P. C. Dorrestein and B. S. Moore, ACS Synth. Biol., 2015, 4, 414-420.

61 Y. Li, Z. Li, K. Yamanaka, Y. Xu, W. Zhang, H. Vlamakis, R. Kolter, B. S. Moore and P. Y. Qian, Sci. Rep., 2015, 5, 9383.

62 Z. R. Li, Y. Li, J. Y. Lai, J. Tang, B. Wang, L. Lu, G. Zhu, X. Wu, Y. Xu and P. Y. Qian, ChemBioChem, 2015, 16, 1715-1719.
63 X. Tang, J. Li, N. Millan-Aguinaga, J. J. Zhang, E. C. O'Neill, J. A. Ugalde, P. R. Jensen, S. M. Mantovani and B. S. Moore, ACS Chem. Biol., 2015, 10, 2841-2849.

64 C. Cano-Prieto, R. Garcia-Salcedo, M. Sanchez-Hidalgo, A. F. Brana, H. P. Fiedler, C. Mendez, J. A. Salas and C. Olano, ChemBioChem, 2015, 16, 1461-1473.

65 N. X. Wu, H. Huang, T. L. Min and H. F. Hu, Acta Biochim. Biophys. Sin., 2017, 49, 1129-1134.

66 J. J. Zhang, X. Tang, M. Zhang, D. Nguyen and B. S. Moore, mBio, 2017, 8.

67 C. B. Larson, M. Crusemann and B. S. Moore, J. Nat. Prod., 2017, 80, 1200-1204.

68 N. C. O. Lee, V. Larionov and N. Kouprina, Nucleic Acids Res., 2015, 43.

69 D. Du, L. Wang, Y. Tian, H. Liu, H. Tan and G. Niu, Sci. Rep., 2015, 5, 8740.

70 O. Bilyk, O. N. Sekurova, S. B. Zotchev and A. Luzhetskyy, PLoS One, 2016, 11, e0158682.

71 C. Paulus, Y. Rebets, J. Zapp, C. Ruckert, J. Kalinowski and A. Luzhetskyy, Front. Microbiol., 2018, 9, 1959.

72 F. Panter, D. Krug, S. Baumann and R. Müller, Chem. Sci., 2018, 9, 4898-4908.

73 Z. Shao, Y. Luo and H. Zhao, Mol. BioSyst., 2011, 7, 10561059.

74 Z. Shao, H. Zhao and H. Zhao, Nucleic Acids Res., 2009, 37, e16.

75 Y. Luo, H. Huang, J. Liang, M. Wang, L. Lu, Z. Shao, R. E. Cobb and H. Zhao, Nat. Commun., 2013, 4, 2894.

76 O. Schimming, F. Fleischhacker, F. I. Nollmann and H. B. Bode, ChemBioChem, 2014, 15, 1290-1294.

77 C. Greunke, E. R. Duell, P. M. D'Agostino, A. Glockle, K. Lamm and T. A. M. Gulder, Metab. Eng., 2018, 47, 334345.

78 P. D'Agostino and T. A. M. Gulder, ACS Synth. Biol., 2018, DOI: 10.1021/acssynbio.8b00151.

79 R. E. Cobb and H. Zhao, Nat. Biotechnol., 2012, 30, 405-406.

80 T. Liu, R. Mazmouz, S. E. Ongley, R. Chau, R. Pickford, J. N. Woodhouse and B. A. Neilan, ACS Chem. Biol., 2017, 12, 2021-2029.

81 Y. Luo, L. Zhang, K. W. Barton and H. Zhao, ACS Synth. Biol., 2015, 4, 1001-1010.

82 S. Li, J. Wang, X. Li, S. Yin, W. Wang and K. Yang, Microb. Cell Fact., 2015, 14, 172.

83 T. Siegl, B. Tokovenko, M. Myronovskyi and A. Luzhetskyy, Metab. Eng., 2013, 19, 98-106.

84 L. Horbal, T. Siegl and A. Luzhetskyy, Sci. Rep., 2018, 8, 491. 85 L. Horbal and A. Luzhetskyy, Metab. Eng., 2016, 37, 11-23. 86 L. Horbal, V. Fedorenko and A. Luzhetskyy, Appl. Microbiol. Biotechnol., 2014, 98, 8641-8655.

87 L. Horbal, F. Marques, S. Nadmid, M. V. Mendes and A. Luzhetskyy, Metab. Eng., 2018, 49, 299-315.

88 C. Bai, Y. Zhang, X. Zhao, Y. Hu, S. Xiang, J. Miao, C. Lou and L. Zhang, Proc. Natl. Acad. Sci. U. S. A., 2015, 112, 12181-12186.

89 C. H. Ji, J. P. Kim and H. S. Kang, ACS Synth. Biol., 2018, 7, 1946-1955. 

T. Paululat, A. Bechthold, L. Petzke and A. Luzhetskyy, J. Biotechnol., 2016, 232, 110-117.

91 L. Li, G. Zheng, J. Chen, M. Ge, W. Jiang and Y. Lu, Metab. Eng., 2017, 40, 80-92.

92 J. P. Gomez-Escribano and M. J. Bibb, Microb. Biotechnol., 2011, 4, 207-215.

93 M. Myronovskyi, B. Rosenkranzer, S. Nadmid, P. Pujic, P. Normand and A. Luzhetskyy, Metab. Eng., 2018, 49, 316-324.

94 B. Bilyk, L. Horbal and A. Luzhetskyy, Microb. Cell Fact., 2017, 16, 5.

95 L. P. Zhu, X. J. Yue, K. Han, Z. F. Li, L. S. Zheng, X. N. Yi, H. L. Wang, Y. M. Zhang and Y. Z. Li, Microb. Cell Fact., 2015, 14, 105.

96 H. R. Zhang, B. A. Boghigian, J. Armando and B. A. Pfeifer, Nat. Prod. Rep., 2011, 28, 125-151.

97 M. Komatsu, T. Uchiyama, S. Omura, D. E. Cane and H. Ikeda, Proc. Natl. Acad. Sci. U. S. A., 2010, 107, 2646-2651.

98 J. Penn, X. Li, A. Whiting, M. Latif, T. Gibson, C. J. Silva, P. Brian, J. Davies, V. Miao, S. K. Wrigley and R. H. Baltz, J. Ind. Microbiol. Biotechnol., 2006, 33, 121-128.

99 N. Zaburannyi, M. Rabyk, B. Ostash, V. Fedorenko and A. Luzhetskyy, BMC Genomics, 2014, 15, 97.

100 S. C. Wenzel and R. Müller, in Industrial Biotechnology, Wiley-VCH Verlag GmbH \& Co. KGaA, 2017, pp. 453-485, DOI: 10.1002/9783527807796.ch12.

101 J. Kumpfmüller, K. Methling, L. Fang, B. A. Pfeifer, M. Lalk and T. Schweder, Appl. Microbiol. Biotechnol., 2016, 100, 1209-1220.

102 A. Loeschcke and S. Thies, Appl. Microbiol. Biotechnol., 2015, 99, 6197-6214.

103 S. C. Wenzel, F. Gross, Y. Zhang, J. Fu, A. F. Stewart and R. Müller, Chem. Biol., 2005, 12, 349-356.

104 Y. Chai, S. Shan, K. J. Weissman, S. Hu, Y. Zhang and R. Müller, Chem. Biol., 2012, 19, 361-371.

105 H. Zhang, L. Fang, M. S. Osburne and B. A. Pfeifer, Methods Mol. Biol., 2016, 1401, 121-134.

106 K. J. Weissman and R. Müller, Nat. Prod. Rep., 2010, 27, 1276-1295.

107 J. Herrmann, A. A. Fayad and R. Müller, Nat. Prod. Rep., 2017, 34, 135-160.

108 S. C. Wenzel and R. Müller, Nat. Prod. Rep., 2009, 26, 13851407.

109 X. Bian, B. Tang, Y. Yu, Q. Tu, F. Gross, H. Wang, A. Li, J. Fu, Y. Shen, Y. Z. Li, A. F. Stewart, G. Zhao, X. Ding, R. Müller and Y. Zhang, ACS Chem. Biol., 2017, 12, 1805-1812. Angew. Chem., Int. Ed. Engl., 2018, DOI: 10.1002/ anie.201802479.

111 X. Wang, H. Zhou, H. Chen, X. Jing, W. Zheng, R. Li, T. Sun, J. Liu, J. Fu, L. Huo, Y. Z. Li, Y. Shen, X. Ding, R. Müller,
90 N. Manderscheid, B. Bilyk, T. Busche, J. Kalinowski,

113 R. K. Singh, S. P. Tiwari, A. K. Rai and T. M. Mohapatra, J. Antibiot., 2011, 64, 401-412.

114 E. J. Kim, J. H. Lee, H. Choi, A. R. Pereira, Y. H. Ban, Y. J. Yoo, E. Kim, J. W. Park, D. H. Sherman, W. H. Gerwick and Y. J. Yoon, Org. Lett., 2012, 14, 5824X. Bian and Y. Zhang, Proc. Natl. Acad. Sci. U. S. A., 2018, 115, E4255-E4263.

112 J. K. Nunnery, E. Mevers and W. H. Gerwick, Curr. Opin. Biotechnol., 2010, 21, 787-793. 5827.

115 S. E. Ongley, X. Bian, Y. Zhang, R. Chau, W. H. Gerwick, R. Müller and B. A. Neilan, ACS Chem. Biol., 2013, 8, 1888-1893.

116 P. Videau, K. N. Wells, A. J. Singh, W. H. Gerwick and B. Philmus, ACS Synth. Biol., 2016, 5, 978-988.

117 B. Wang, C. Eckert, P. C. Maness and J. P. Yu, ACS Synth. Biol., 2018, 7, 276-286.

118 A. Guljamow, M. Kreische, K. Ishida, A. Liaimer, B. Altermark, L. Bahr, C. Hertweck, R. Ehwald and E. Dittmann, Appl. Environ. Microbiol., 2017, DOI: 10.1128/aem.01510-17.

119 J. Roulet, A. Taton, J. W. Golden, A. Arabolaza, M. D. Burkart and H. Gramajo, Metab. Eng., 2018, 49, 94104.

120 M. Komatsu, K. Komatsu, H. Koiwai, Y. Yamada, I. Kozone, M. Izumikawa, J. Hashimoto, M. Takagi, S. Omura, K. Shinya, D. E. Cane and H. Ikeda, ACS Synth. Biol., 2013, 2, 384396.

121 K. R. Choi, J. S. Cho, I. J. Cho, D. Park and S. Y. Lee, Metab. Eng., 2018, 47, 463-474.

122 P. I. Nikel and V. de Lorenzo, Metab. Eng., 2018, DOI: 10.1016/j.ymben.2018.05.005.

123 M. Baumgart, S. Unthan, R. Kloss, A. Radek, T. Polen, N. Tenhaef, M. F. Müller, A. Kuberl, D. Siebert, N. Bruhl, K. Marin, S. Hans, R. Kramer, M. Bott, J. Kalinowski, W. Wiechert, G. Seibold, J. Frunzke, C. Ruckert, V. F. Wendisch and S. Noack, ACS Synth. Biol., 2018, 7, 132-144.

124 Y. Luo, B. Z. Li, D. Liu, L. Zhang, Y. Chen, B. Jia, B. X. Zeng, H. Zhao and Y. J. Yuan, Chem. Soc. Rev., 2015, 44, 52655290.

125 R. Liu, Z. Deng and T. Liu, Metab. Eng., 2018, DOI: 10.1016/ j.ymben.2018.05.015.

126 R. H. Baltz, J. Ind. Microbiol. Biotechnol., 2010, 37, 759-772.

127 S. Yin, Z. Li, X. Wang, H. Wang, X. Jia, G. Ai, Z. Bai, M. Shi, F. Yuan, T. Liu, W. Wang and K. Yang, Appl. Microbiol. Biotechnol., 2016, 100, 10563-10572.

128 R. Novakova, L. E. Nunez, D. Homerova, R. Knirschova, L. Feckova, B. Rezuchova, B. Sevcikova, N. Menendez, F. Moris, J. Cortes and J. Kormanec, Appl. Microbiol. Biotechnol., 2018, 102, 857-869.

129 G. Y. Tan, K. Deng, X. Liu, H. Tao, Y. Chang, J. Chen, K. Chen, Z. Sheng, Z. Deng and T. Liu, ACS Synth. Biol., 2017, 6, 995-1005.

130 J. Huang, Z. Yu, M. H. Li, J. D. Wang, H. Bai, J. Zhou and Y. G. Zheng, Appl. Environ. Microbiol., 2016, 82, 5603-5611.

131 T. F. Schaberle, A. Schiefer, A. Schmitz, G. M. Konig, A. Hoerauf and K. Pfarr, Int. J. Med. Microbiol., 2014, 304, 72-78.

132 H. Sucipto, D. Pogorevc, E. Luxenburger, S. C. Wenzel and R. Müller, Metab. Eng., 2017, 44, 160-170. 
133 Q. Tu, J. Herrmann, S. Hu, R. Raju, X. Bian, Y. Zhang and R. Müller, Sci. Rep., 2016, 6, 21066.

134 K. Gemperlein, G. Zipf, H. S. Bernauer, R. Müller and S. C. Wenzel, Metab. Eng., 2016, 33, 98-108.

135 Y. Sun, Z. Feng, T. Tomura, A. Suzuki, S. Miyano, T. Tsuge, H. Mori, J. W. Suh, T. Iizuka, R. Fudou and M. Ojika, Sci. Rep., 2016, 6, 22091.

136 X. J. Yue, X. W. Cui, Z. Zhang, W. F. Hu, Z. F. Li, Y. M. Zhang and Y. Z. Li, Appl. Microbiol. Biotechnol., 2018, 102, 55995610.

137 Y. J. Yang, Y. Wang, Z. F. Li, Y. Gong, P. Zhang, W. C. Hu, D. H. Sheng and Y. Z. Li, Microb. Cell Fact., 2017, 16, 142.

138 R. Peng, Y. Wang, W. W. Feng, X. J. Yue, J. H. Chen, X. Z. Hu, Z. F. Li, D. H. Sheng, Y. M. Zhang and Y. Z. Li, Microb. Cell Fact., 2018, 17, 15.

139 X. J. Yue, X. W. Cui, Z. Zhang, W. F. Hu, Z. F. Li, Y. M. Zhang and Y. Z. Li, Appl. Microbiol. Biotechnol., 2018, 102, 55995610.

140 C. Liu, F. Yu, Q. Liu, X. Bian, S. Hu, H. Yang, Y. Yin, Y. Li, Y. Shen, L. Xia, Q. Tu and Y. Zhang, FEMS Microbiol. Lett., 2018, 365.

141 V. Bischoff, S. J. Cookson, S. Wu and W. R. Scheible, J. Exp. Bot., 2009, 60, 955-965.

142 W. R. Scheible, B. Fry, A. Kochevenko, D. Schindelasch, L. Zimmerli, S. Somerville, R. Loria and C. R. Somerville, Plant Cell, 2003, 15, 1781-1794.

143 R. R. King and L. A. Calhoun, Phytochemistry, 2009, 70, 833841.

144 G. D. Jiang, Y. C. Zhang, M. M. Powell, P. L. Zhang, R. Zuo, Y. Zhang, D. Kallifidas, A. M. Tieu, H. Luesch, R. Loria and Y. S. Ding, Appl. Environ. Microbiol., 2018, 84.

145 M. Winn, D. Francis and J. Micklefield, Angew. Chem., Int. Ed. Engl., 2018, 57, 6830-6833.

146 K. Blin, M. H. Medema, D. Kazempour, M. A. Fischbach, R. Breitling, E. Takano and T. Weber, Nucleic Acids Res., 2013, 41, W204-W212.

147 C. Osswald, N. Zaburannyi, C. Burgard, T. Hoffmann, S. C. Wenzel and R. Müller, J. Biotechnol., 2014, 191, 54-63. 148 B. Julien, J. Bacteriol., 2003, 185, 6325-6330.

149 J. A. Blodgett, D. C. Oh, S. Cao, C. R. Currie, R. Kolter and J. Clardy, Proc. Natl. Acad. Sci. U. S. A., 2010, 107, 1169211697.

150 J. Antosch, F. Schaefers and T. A. Gulder, Angew. Chem., Int. Ed. Engl., 2014, 53, 3011-3014.

151 Y. Liu, H. Wang, R. Song, J. Chen, T. Li, Y. Li, L. Du and Y. Shen, Org. Lett., 2018, 20, 3504-3508.

152 S. Saha, W. Zhang, G. Zhang, Y. Zhu, Y. Chen, W. Liu, C. Yuan, Q. Zhang, H. Zhang, L. Zhang, W. Zhang and C. Zhang, Chem. Sci., 2017, 8, 1607-1612.

153 Y. Qi, E. Ding and J. A. V. Blodgett, ACS Synth. Biol., 2018, 7, 357-362.

154 F. Lombo, B. Pfeifer, T. Leaf, S. Ou, Y. S. Kim, D. E. Cane, P. Licari and C. Khosla, Biotechnol. Prog., 2001, 17, 612-617.

155 D. Cao, K. Polyak, M. K. Halushka, H. Nassar, N. Kouprina, C. Iacobuzio-Donahue, X. Wu, S. Sukumar, J. Hicks, A. De Marzo and P. Argani, Breast Cancer Res., 2008, 10, R91.
156 X. Xu, H. Zhou, Y. Liu, X. Liu, J. Fu, A. Li, Y. Z. Li, Y. Shen, X. Bian and Y. Zhang, J. Nat. Prod., 2018, 81, 1060-1064.

157 Y. Shi, Z. Jiang, X. Li, L. Zuo, X. Lei, L. Yu, L. Wu, J. Jiang and B. Hong, Acta Pharm. Sin. B, 2018, 8, 283-294.

158 M. Crusemann, R. Reher, I. Schamari, A. O. Brachmann, T. Ohbayashi, M. Kuschak, D. Malfacini, A. Seidinger, M. Pinto-Carbo, R. Richarz, T. Reuter, S. Kehraus, A. Hallab, M. Attwood, H. B. Schioth, P. Mergaert, Y. Kikuchi, T. F. Schaberle, E. Kostenis, D. Wenzel, C. E. Müller, J. Piel, A. Carlier, L. Eberl and G. M. Konig, Angew. Chem., Int. Ed. Engl., 2018, 57, 836-840.

159 T. Fukuda, E. D. Miller, B. R. Clark, A. Alnauman, C. D. Murphy, P. R. Jensen and W. Fenical, J. Nat. Prod., 2011, 74, 1773-1778.

160 L. Linares-Otoya, V. Linares-Otoya, L. Armas-Mantilla, C. Blanco-Olano, M. Crusemann, M. L. Ganoza-Yupanqui, J. Campos-Florian, G. M. Konig and T. F. Schaberle, Mar. Drugs, 2017, 15.

161 P. Bekiesch, P. Basitta and A. K. Apel, Arch. Pharm., 2016, 349, 594-601.

162 M. Xu, Y. Wang, Z. Zhao, G. Gao, S. X. Huang, Q. Kang, X. He, S. Lin, X. Pang, Z. Deng and M. Tao, Appl. Environ. Microbiol., 2016, 82, 5795-5805.

163 C. Yang, C. Huang, W. Zhang, Y. Zhu and C. Zhang, Org. Lett., 2015, 17, 5324-5327.

164 L. Hou, H. Huang, H. Li, S. Wang, J. Ju and W. Li, Microb. Cell Fact., 2018, 17, 61.

165 Y. Li, H. Wang, Y. Liu, Y. Jiao, S. Li, Y. Shen and L. Du, Angew. Chem., Int. Ed. Engl., 2018, 57, 6221-6225.

166 Y. Li, H. Chen, Y. Ding, Y. Xie, H. Wang, R. L. Cerny, Y. Shen and L. Du, Angew. Chem., Int. Ed. Engl., 2014, 53, 7524-7530.

167 G. Zhang, W. Zhang, S. Saha and C. Zhang, Curr. Top. Med. Chem., 2016, 16, 1727-1739.

168 T. Thongkongkaew, W. Ding, E. Bratovanov, E. Oueis, A. A. M. A. Garci, N. Zaburannyi, K. Harmrolfs, Y. Zhang, K. Scherlach, R. Müller and C. Hertweck, ACS Chem. Biol., 2018, 13, 1370-1379.

169 H. B. Park, C. E. Perez, K. W. Barber, J. Rinehart and J. M. Crawford, eLife, 2017, 6.

170 I. G. U. Pait, S. Kitani, F. W. Roslan, D. Ulanova, M. Arai, H. Ikeda and T. Nihira, J. Ind. Microbiol. Biotechnol., 2018, 45, 77-87.

171 X. Cai, V. L. Challinor, L. Zhao, D. Reimer, H. Adihou, P. Grun, M. Kaiser and H. B. Bode, Org. Lett., 2017, 19, 806-809.

172 T. Kawahara, M. Izumikawa, I. Kozone, J. Hashimoto, N. Kagaya, H. Koiwai, M. Komatsu, M. Fujie, N. Sato, H. Ikeda and K. Shin-Ya, J. Nat. Prod., 2018, 81, 264-269.

173 H. Wang, X. Bian, L. Xia, X. Ding, R. Müller, Y. Zhang, J. Fu and A. F. Stewart, Nucleic Acids Res., 2014, 42, e37.

174 X. F. Cai, S. Nowak, F. Wesche, I. Bischoff, M. Kaiser, R. Furst and H. B. Bode, Nat. Chem., 2017, 9, 379-386.

175 K. A. J. Bozhuyuk, F. Fleischhacker, A. Linck, F. Wesche, A. Tietze, C. P. Niesert and H. B. Bode, Nat. Chem., 2018, 10, 275-281.

176 F. Kopp and M. A. Marahiel, Nat. Prod. Rep., 2007, 24, 735749. 
177 F. Yan, C. Burgard, A. Popoff, N. Zaburannyi, G. Zipf, J. Maier, H. S. Bernauer, S. C. Wenzel and R. Müller, Chem. Sci., 2018, 9, 7510-7519.

178 S. Cleto and T. K. Lu, mBio, 2017, 8.

179 M. Lopatniuk, M. Myronovskyi and A. Luzhetskyy, ACS Chem. Biol., 2017, 12, 2362-2370.

180 V. Torsvik, J. Goksoyr and F. L. Daae, Appl. Environ. Microbiol., 1990, 56, 782-787.

181 J. Handelsman, M. R. Rondon, S. F. Brady, J. Clardy and R. M. Goodman, Chem. Biol., 1998, 5, R245-R249.

182 F. Y. Chang and S. F. Brady, ChemBioChem, 2014, 15, 815821.

183 F. Y. Chang, M. A. Ternei, P. Y. Calle and S. F. Brady, J. Am. Chem. Soc., 2015, 137, 6044-6052.

184 B. M. Hover, S. H. Kim, M. Katz, Z. Charlop-Powers, J. G. Owen, M. A. Ternei, J. Maniko, A. B. Estrela, H. Molina, S. Park, D. S. Perlin and S. F. Brady, Nat. Microbiol., 2018, 3, 415-422.

185 H. A. Iqbal, L. Low-Beinart, J. U. Obiajulu and S. F. Brady, J. Am. Chem. Soc., 2016, 138, 9341-9344.

186 H. S. Kang and S. F. Brady, Angew. Chem., Int. Ed. Engl,, 2013, 52, 11063-11067.

187 H. S. Kang and S. F. Brady, ACS Chem. Biol., 2014, 9, 12671272.

188 J. G. Owen, Z. Charlop-Powers, A. G. Smith, M. A. Ternei, P. Y. Calle, B. V. Reddy, D. Montiel and S. F. Brady, Proc. Natl. Acad. Sci. U. S. A., 2015, 112, 4221-4226.

189 S. Nasrin, S. Ganji, K. S. Kakirde, M. R. Jacob, M. Wang, R. R. Ravu, P. A. Cobine, I. A. Khan, C. C. Wu, D. A. Mead, X. C. Li and M. R. Liles, J. Nat. Prod., 2018, 81, 1321-1332.

190 V. Agarwal, J. M. Blanton, S. Podell, A. Taton, M. A. Schorn, J. Busch, Z. Lin, E. W. Schmidt, P. R. Jensen, V. J. Paul, J. S. Biggs, J. W. Golden, E. E. Allen and B. S. Moore, Nat. Chem. Biol., 2017, 13, 537-543.

191 T. E. Smith, C. D. Pond, E. Pierce, Z. P. Harmer, J. Kwan, M. M. Zachariah, M. K. Harper, T. P. Wyche, T. K. Matainaho, T. S. Bugni, L. R. Barrows, C. M. Ireland and E. W. Schmidt, Nat. Chem. Biol., 2018, 14, 179-185.

192 C. J. Guo, F. Y. Chang, T. P. Wyche, K. M. Backus, T. M. Acker, M. Funabashi, M. Taketani, M. S. Donia, S. Nayfach, K. S. Pollard, C. S. Craik, B. F. Cravatt,
J. Clardy, C. A. Voigt and M. A. Fischbach, Cell, 2017, 168, 517-+.

193 E. J. N. Helfrich, C. M. Vogel, R. Ueoka, M. Schafer, F. Ryffel, D. B. Muller, S. Probst, M. Kreuzer, J. Piel and J. A. Vorholt, Nat. Microbiol., 2018, 3, 909-919.

194 J. K. Patel and G. Archana, Appl. Soil Ecol., 2018, 124, 34-44. 195 J. J. Jimenez, D. B. Diep, J. Borrero, L. Gutiez, S. Arbulu, I. F. Nes, C. Herranz, L. M. Cintas and P. E. Hernandez, Microb. Cell Fact., 2015, 14, 166.

196 G. M. Daba, N. Ishibashi, T. Zendo and K. Sonomoto, J. Appl. Microbiol., 2017, 123, 1124-1132.

197 J. L. Shang, Z. C. Zhang, X. Y. Yin, M. Chen, F. H. Hao, K. Wang, J. L. Feng, H. F. Xu, Y. C. Yin, H. R. Tang and B. S. Qiu, Environ. Microbiol., 2018, 20, 200-213.

198 M. Katoch, R. Mazmouz, R. Chau, L. A. Pearson, R. Pickford and B. A. Neilan, Appl. Environ. Microbiol., 2016, 82, 61676173.

199 J. Borrero, E. Kelly, P. M. O'Connor, P. Kelleher, C. Scully, P. D. Cotter, J. Mahony and D. van Sinderen, Appl. Environ. Microbiol., 2018, 84.

200 M. Izawa, T. Kawasaki and Y. Hayakawa, Appl. Environ. Microbiol., 2013, 79, 7110-7113.

201 M. Izawa, S. Nagamine, H. Aoki and Y. Hayakawa, J. Gen. Appl. Microbiol., 2018, 64, 50-53.

202 L. Yi, Y. Dang, J. Wu, L. Zhang, X. Liu, B. Liu, Y. Zhou and X. Lu, J. Dairy Sci., 2016, 99, 7002-7015.

203 N. M. Vior, R. Lacret, G. Chandra, S. Dorai-Raj, M. Trick and A. W. Truman, Appl. Environ. Microbiol., 2018, 84.

204 J. Mevaere, C. Goulard, O. Schneider, O. N. Sekurova, H. Y. Ma, S. Zirah, C. Afonso, S. Rebuffat, S. B. Zotchev and Y. Y. Li, Sci. Rep., 2018, 8.

205 C. Su, X. Zhao, R. Qiu and L. Tang, Pharm. Biol., 2015, 53, 269-274.

206 H. Wolff and H. B. Bode, PLoS One, 2018, 13, e0194297.

207 A. von Tesmar, M. Hoffmann, A. Abou Fayad, S. Huttel, V. Schmitt, J. Herrmann and R. Müller, ACS Chem. Biol., 2018, 13, 812-819.

208 L. Frattaruolo, R. Lacret, A. R. Cappello and A. W. Truman, ACS Chem. Biol., 2017, 12, 2815-2822.

209 H. S. Kang and S. F. Brady, J. Am. Chem. Soc., 2014, 136, 18111-18119. 